\title{
Screening of lactic acid bacteria and yeast strains to select adapted anti-fungal co-cultures for cocoa bean fermentation
}

Edwina Romanens, Susette Freimüller Leischtfeld, Andrea Volland, Marc J. A. Stevens, Ursina Krähenmann, Désirée Isele, Birgit Fischer, Leo Meile, Susanne Miescher Schwenninger

This article has been published in International Journal of Food Microbiology, 290, 262-272, 2019. doi: 10.1016/j.ijfoodmicro.2018.10.001 


\section{Abstract}

Contamination with filamentous fungi during cocoa bean fermentation and drying reduces the quality of cocoa beans and poses a health risk for consumers due to the potential accumulation of mycotoxins. The aim of this study was to develop anti-fungal lactic acid bacteria (LAB)yeast co-cultures by selecting anti-fungal strains best adapted to the cocoa bean fermentation process from $362 \mathrm{LAB}$ and 384 yeast strains isolated from cocoa bean post-harvest processes. The applied multiphasic screening approach included anti-fungal activity tests in vitro and in vivo and assessment of the carbon metabolism and stress tolerance of the anti-fungal strains in cocoa pulp simulation medium. The anti-fungal strains, Lactobacillus fermentum M017, Lactobacillus fermentum 223, Hanseniaspora opuntiae H17, and Saccharomyces cerevisiae H290, were selected based on their high inhibition capacity and their well-adapted metabolism. Up to seven filamentous fungal strains of the genera Aspergillus, Penicillium, and Gibberella were inhibited by 63 and 75\% of the maximal inhibition zone by M017 and 223, respectively, and by 25 and $31 \%$ by the strains $\mathrm{H} 17$ and H290, respectively. Both Lb. fermentum strains converted the medium's glucose, fructose, and citric acid into 20.4-23.0 g/1 of mannitol, 3.9$6.2 \mathrm{~g} / 1$ acetic acid, and 8.6-10.3 g/l lactic acid, whereas the two yeast strains metabolized glucose and fructose to produce 7.4-18.4 g/l of ethanol. The $L b$. fermentum strains were further characterized as ethanol, acetic acid, and temperature tolerant and both yeast strains as ethanol and lactic acid tolerant. Finally, the anti-fungal in vivo assays revealed that the two Lb. fermentum strains completely inhibited growth of the citrinin-producing strain, P. citrinum S005, and the potentially fumonisin-producing strain, G. moniliformis S003, on the surface of cocoa beans. All four selected anti-fungal strains, i.e. Lb. fermentum M017, Lb. fermentum 223, H. opuntiae H17, and Sacc. cerevisiae H290, inhibited at 51-95\% growth of aflatoxin-producer A. flavus S075 as single cultures while preventing its growth by $100 \%$ when combined into four co-cultures, each composed of a $L b$. fermentum strain and one of the two yeast strains. As a conclusion, these four LAB-yeast co-cultures are recommended for future applications to limit the growth of filamentous fungi and the concomitant mycotoxin production during the fermentation of cocoa beans. 


\section{Introduction}

With a global annual production estimated at 4.7 million tonnes in the crop season 2016/2017, cocoa is the main ingredient for chocolate production (Beg et al., 2017; ICCO, 2017b). The first step in cocoa production is a spontaneous fermentation process which is necessary to remove the pulp around the beans allowing metabolites to enter the cotyledon and turning the astringent and bitter flavours into precursors of typical chocolate aroma and flavours (De Vuyst and Weckx, 2016). During the fermentation, there is a well-defined microbial succession of yeasts, lactic acid bacteria (LAB), and acetic acid bacteria (AAB) (Pereira et al., 2016), however, filamentous fungi can also grow, mainly in the well-aerated and cold outer layers of the fermentation mass and during the drying process (Nielsen et al., 2013; Schwan and Fleet, 2014). Contamination with filamentous fungi has been associated with internal development of mould, off-flavours, increased free fatty acid levels, and the production of mycotoxins (Nielsen et al., 2013). Mycotoxins reported to be of major significance in cocoa include aflatoxin B1, associated with Aspergillus flavus and Aspergillus parasiticus, ochratoxin A (OTA), commonly biosynthesized by Penicillium spp. and Aspergillus ochraceus, fumonisin, produced by Fusarium spp., and citrinin produced by Penicillium citrinum (Badrie et al., 2015; Copetti et al., 2014; Nielsen et al., 2013; Sánchez-Hervás et al., 2008). Out of 85 cocoa samples collected during sun drying and 65 samples collected during storage in Brazil, 38\% and 32\%, respectively, were infected by $A$. flavus and $24 \%$ and $14 \%$, respectively, by A.parasiticus (Copetti et al., 2011). Aflatoxin B1 was detected in 25\% of Brazilian cocoa bean samples at levels of up to $5.9 \mu \mathrm{g} / \mathrm{kg}$ (Maciel et al., 2018) and cocoa products sampled in Canada contained up to $2.6 \mu \mathrm{g} / \mathrm{kg}$ aflatoxin B1 (Turcotte et al., 2013). The European Community (EC) limited the level of aflatoxin B1 in nuts intended for direct human consumption or use as an ingredient in food to $2.0 \mu \mathrm{g} / \mathrm{kg}$, however, no limit was set for cocoa beans.

Microorganisms are of great interest as biocontrol agents to reduce mould growth and the concomitant contamination with mycotoxins. LAB have been intensively studied as biocontrol agents, such as to retard or eliminate fungal growth based on the production of a wide spectrum of antimicrobial compounds in form of organic acids, low molecular weight compounds, hydroxyl fatty acids, or proteinaceous metabolites, e.g. bacteriocins (Peyer et al., 2016). Furthermore, yeasts with antagonistic effects against filamentous fungi are frequently used as biopreservatives to control postharvest diseases on fruits (Spadaro and Droby, 2016). As reviewed by Pereira et al. (2016), efforts in recent years have been made to develop starter 
cultures for the cocoa bean fermentation, comprising different species of LAB, yeasts, and/or AAB. LAB and yeast strains were recently screened for their ability to inhibit OTA-producing filamentous fungi (de Souza et al., 2017; Essia Ngang et al., 2015; Fossi et al., 2016) with the aim of overcoming problems of mould contamination and mycotoxin accumulation during the cocoa bean fermentation and thereby obtaining a safer end product. However to date, no study has reported anti-fungal cultures to control aflatoxin-, citrinin-, or fumonisin-producing fungal strains.

The aim of this study was to develop well-adapted anti-fungal LAB-yeast co-cultures for the cocoa bean fermentation with a focus on inhibiting aflatoxin-, citrinin-, and fumonisinproducing fungal species. In a multiphasic approach, a large number of cocoa-derived LAB and yeast strains was screened for anti-fungal activity in vitro and/or in vivo against filamentous fungal strains producing or potentially producing aflatoxin, citrinin, and fumonisin. To provide cultures with a high survival potential during cocoa bean fermentation conditions, the antifungal strains' tolerance toward acid, ethanol, and heat stress and their fermentative potential were assessed in vitro.

\section{Material and methods}

\section{Microbial strains and filamentous fungal spore production}

The microbial strains used in the present study, i.e. 362 lactic acid bacteria (LAB) and 384 yeasts, had been isolated from cocoa bean fermentation and/or drying processes in Honduras (Romanens et al., 2018; unpublished data), Bolivia and Brazil (Miescher Schwenninger et al., 2016), and Switzerland (unpublished data). The seven filamentous fungi used as indicator strains originated from Honduras and were retrieved from mould-infested dry cocoa beans, except for Aspergillus candidus S010 that was isolated from the wood of a fermentation box and Aspergillus nidulans S049 that originated from an on-farm fermentation. Growth of strains for proliferation and maintenance in the laboratory were also described therein. Additionally, antibiotic susceptibility for selected LAB strains was tested by disc diffusion tests according to NCCLS guidelines (Patel et al., 2015). LAB and yeast strains were identified with MALDITOF MS prior to this study as described in the above-mentioned publications. In the present work, strains with a MALDI-TOF identification score of 1.700-1.999 were listed according to their identification at genus level and strains with a score of $\geq 2.000$ with their identification at 
species level. LAB strains with a score of $<1.700$ were listed as LAB based on their catalasenegative and oxidase-negative behaviour. When LAB and yeast strains were additionally identified through genome sequencing and PCR, respectively, in the present study (section 2.3.7), their identities refer to these two methods. Filamentous fungal strains were identified previously by sequencing PCR amplicons of the ITS region as described below in section 2.3.7. Throughout this study, fungal species were named according to the taxonomy database of the National Center for Biotechnology Information (NCBI; https://www.ncbi.nlm.nih.gov/). Microbial strains listed by name, i.e. 26 LAB (Supplementary Tab. S1), 63 yeasts (Supplementary Tab. S2), and the 7 filamentous fungi mentioned below, are stored in the culture collection of Switzerland (CCOS, Wädenswil, Switzerland).

Spore suspensions of the filamentous fungal indicator strains Aspergillus candidus S010, Aspergillus flavus S075, Aspergillus nidulans S049, Aspergillus tamarii S078, Aspergillus versicolor S085, Gibberella moniliformis S003, and Penicillium citrinum S005, were prepared for anti-fungal inhibition tests in vitro and in vivo. The filamentous fungi were therefore incubated for 5-7 days at $25{ }^{\circ} \mathrm{C}$ on Malt Extract Agar (MEA) $(1.8 \%$ [w/v] malt extract [Biolife], $1.8 \%[\mathrm{w} / \mathrm{v}]$ bacteriological agar), and the spores were harvested with $0.15 \%(\mathrm{w} / \mathrm{v})$ buffered peptone water (Merck, Darmstadt, Germany). The spore suspensions were mixed with glycerol (AppliChem, Darmstadt, Germany) to a final glycerol concentration of $44 \%(\mathrm{v} / \mathrm{v})$ and stored at $-20{ }^{\circ} \mathrm{C}$. Before usage, the spore concentration was determined by plating appropriate dilutions on MEA.

\section{Cocoa pulp simulation medium}

The cocoa pulp simulation medium developed by Lefeber et al. (2010) was slightly adapted for the experiments with LAB and yeast test strains and was therefore named modified Cocoa Pulp Simulation Medium (mCPSM). Soy peptone was substituted with the more economical meat peptone in view of using mCPSM for industrial-scale culture production. The medium contained 2.5\% (w/v) fructose (Carl Roth, Karlsruhe, Germany), 2.5\% (w/v) glucose (SigmaAldrich, Saint Louis, US), 1\% (w/v) citric acid (Carl Roth), 0.5\% (w/v) yeast extract (SigmaAldrich), 0.5\% (w/v) meat peptone (Organotechnie, La Courneuve, France), 0.05\% (w/v) magnesium sulphate-heptahydrate (Carl Roth), 0.02\% (w/v) manganese sulphate-monohydrate (Sigma-Aldrich), $0.1 \%$ (v/v) tween 80 (Sigma-Aldrich) (vol/vol), and $1.5 \%$ (w/v) bacteriological agar (Biolife, Milan, Italy) when preparing solid medium. To prevent the Maillard reaction, glucose, fructose, and citric acid were sterilized separately and added to the 
remaining components after sterilization. The $\mathrm{pH}$ of the medium was adjusted with $10 \mathrm{M} \mathrm{NaOH}$ solution to 4.0 for liquid medium and to 4.5 when preparing agar plates.

\section{Mycotoxin production of filamentous fungal strains}

The potential of $A$. flavus $\mathrm{S} 075$ to produce aflatoxin was assessed genotypically by detecting the genes aflD, aflO and aflP by PCR (Scherm et al., 2005) and phenotypically on yeast extract sucrose agar supplemented with $0.3 \% \quad \beta$-W7M $\quad 1.8$-cyclodextrin and $0.6 \%$ sodium desoxycholate (YCSD) (Jaimez Ordaz et al., 2003). The potential of G. moniliformis S003 to produce fumonisin was determined genotypically by assessing the presence of the genes fum 1 and fum 19 by PCR (López-Errasquín et al., 2007), and P. citrinum S005 was tested phenotypically for citrinin production on Coconut Cream Agar (CCA) (Mohamed et al., 2013). Further, A. flavus S075, G. moniliformis S003, and P. citrinum S005 were tested in vivo for mycotoxin production by inoculating samples of $80 \mathrm{~g}$ of cocoa beans with $6 \mathrm{log} \mathrm{CFU} / \mathrm{g}$ of spores and sending the mouldy bean samples, after 11 days of incubation at $25^{\circ} \mathrm{C}$, to the contract laboratory UFAG Laboratorien (Sursee, Switzerland) for mycotoxin analyses.

\section{Inhibition of filamentous fungi by LAB and yeast strains}

\section{In vitro anti-fungal agar spot assay}

A modified version of the overlay method of Hassan and Bullerman (2008) was used to assess in vitro anti-fungal activity of LAB and yeast strains. $\mathrm{LAB}$ were inoculated with sterile toothpicks onto De Man-Rogosa-Sharpe (MRS; Biolife) agar buffered with $0.1 \mathrm{M} \mathrm{KH}_{2} \mathrm{PO}_{4}-$ $\mathrm{K}_{2} \mathrm{HPO}_{4}$ (pH 6.4) or mCPSM agar and incubated anaerobically at $37{ }^{\circ} \mathrm{C}$ for 1 day. The resulting agar plates containing the LAB colonies and non-inoculated MEA or mCPSM agar plates for assays with yeasts were overlaid with $10 \mathrm{ml}$ soft agar supplemented with $4 \log \mathrm{CFU} / \mathrm{ml}$ filamentous fungal spores and tempered at $50{ }^{\circ} \mathrm{C}$. MEA soft agar with $0.7 \%(\mathrm{w} / \mathrm{v})$ agar was used for LAB agar spot assays and MEA and mCPSM soft agar containing 1.0\% (w/v) agar was used in yeast agar spot assays to overlay MEA and mCPSM agar plates, respectively. Yeast strains were inoculated directly onto the soft-agar layer using sterile toothpicks.

The agar spot assay plates of LAB and yeasts were then incubated aerobically at $25^{\circ} \mathrm{C}$ for 3 days, except for plates with spores of $G$. moniliformis S003 that were incubated at room temperature $\left(19-23{ }^{\circ} \mathrm{C}\right)$ and in daylight. Excessive growth of A. flavus $\mathrm{S} 075$ and A. tamarii S078 was slowed down by interrupting the incubation after $30 \mathrm{~h}$ by a phase of $15 \mathrm{~h}$ at $4{ }^{\circ} \mathrm{C}$ before placing the plates back in the incubator. 
The inhibition of filamentous fungi was evaluated visually after 3 days of incubation, except for yeast on mCPSM, for which the zones of inhibition were evaluated after 3, 4, 5, and 7 days and the highest value from the different days was used. To determine a score of inhibition (SI), the distance from the edge of the $\mathrm{LAB}$ or the yeast colony to the outer edge of the zone of inhibition was assessed, along with the transparency of the inhibition zone. In terms of LAB, SI were determined by rating the inhibition zones on a scale from 0 to 4, allowing half points: (0) no inhibition, (1) spore formation delayed but no clear zone, (2), fungal growth delayed with a small clear zone around the colony $(<2 \mathrm{~mm})$, (3) fungal growth delayed with a medium-sized clear zone around the colony (2-4 mm), and (4) fungal growth delayed with a large clear zone around colonies $(>4 \mathrm{~mm})$. In the case of yeasts, the zones of inhibition were rated on a scale: (0) no inhibition, (1) spore formation delayed with a small turbid zone around the colony $(<1 \mathrm{~mm})$, and (2) spore formation delayed with a medium-sized turbid zone around the colony $(2-3 \mathrm{~mm})$. These scores resulting from yeast agar spot assays were multiplied by a factor of 2 if the inhibition zone was semi-clear and a factor of 3 for clear inhibition zones, resulting in SI values of between 0 and 6 for yeasts. Average scores of inhibition (ØSI) were calculated per LAB and yeast strain on MRS and mCPSM or MEA and mCPSM, respectively, averaging the scores of inhibition (SI) obtained for the different tested filamentous fungal indicator strains.

\section{In vivo anti-fungal assay on cocoa beans}

The in vivo anti-fungal activity of LAB and yeast strains was assessed directly on cocoa beans. $\mathrm{LAB}$ and yeast strains were propagated once in MCPSM and inoculated in mCPSM broth, followed by incubation at $37^{\circ} \mathrm{C}$ for $15 \mathrm{~h}$ for $\mathrm{LAB}$ and at $25^{\circ} \mathrm{C}$ for $21 \mathrm{~h}$ for yeast. After centrifugation, cell suspensions at defined concentrations were prepared by resuspending the pellet in $0.15 \%(\mathrm{w} / \mathrm{v})$ buffered peptone water. Twenty grams of cocoa pulp bean mass, previously extracted under sterile conditions from ripe cocoa fruits and stored at $-20{ }^{\circ} \mathrm{C}$, was inoculated with fungal spores at $6 \log \mathrm{CFU} / \mathrm{g}, \mathrm{LAB}$ cells at $6 \log \mathrm{CFU} / \mathrm{g}$ and/or yeast cells at 3 $\log \mathrm{CFU} / \mathrm{g}$ in single culture tests and at $2 \mathrm{log} \mathrm{CFU} / \mathrm{g}$ in LAB-yeast co-culture tests. The inoculated beans were filled into $10-\mathrm{ml}$ petri dishes and incubated for 4 days at $30{ }^{\circ} \mathrm{C}$. After this first incubation step, beans from LAB single-culture trials were incubated for 6-7 days at room temperature $\left(19-23^{\circ} \mathrm{C}\right)$ in a second incubation step. For the second incubation step of yeast and LAByeast co-culture assays, beans were placed on Dichloran Rose Bengal Chloramphenicol (DRBC) agar (Sigma-Aldrich, Saint Louis, USA) as described by Pitt and Hocking (2009) and incubated at $25^{\circ} \mathrm{C}$ for 10 days. The inhibition of filamentous fungal growth was evaluated visually as share of non-infested bean surface relative to the total surface at the end of the first and the second 
incubation steps. Furthermore, beans inoculated with only LAB and/or yeast cells, with filamentous fungal spores alone, or without any added cells or spores were incubated and evaluated in an analogous manner.

At the end of the first phase of incubation, the anti-fungal strains were enumerated on the surface of beans that were only inoculated with cells of LAB and/or yeast strains. Two beans with adhering pulp were mixed manually for $1 \mathrm{~min}$ with the same weight of dilution solution $(0.1 \%[\mathrm{w} / \mathrm{v}]$ bacteriological peptone [Biolife], $0.85 \%[\mathrm{w} / \mathrm{v}]$ sodium chloride) to obtain a uniform homogenate of pulp and dilution solution. After decimal dilution steps, $20 \mu 1$ of up to four dilutions were applied on one section of an agar plate followed by holding the plate slantwise for the drops to elongate. The enumeration of yeasts was performed on Yeast Extract Glucose Chloramphenicol (YGC) agar (Biolife) after aerobic incubation at $25^{\circ} \mathrm{C}$ for 3 days and the counting of LAB on MRS agar after anaerobic incubation at $37^{\circ} \mathrm{C}$ for $2-3$ days. The pulp $\mathrm{pH}$ was measured with a $\mathrm{pH}$ meter ( $\mathrm{pH}-$ Meter 761 Calimatic, Merck) in the pulp-dilution solution homogenate mentioned above.

\section{Measuring substrates and metabolites in LAB and yeast fermentation filtrates}

Starting from pure colonies on mCPSM agar plates, precultures were prepared by inoculation in mCPSM broth and incubation at $37^{\circ} \mathrm{C}$ for $19 \mathrm{~h}$ for LAB and at $25^{\circ} \mathrm{C}$ for $21 \mathrm{~h}$ for yeast. For the fermentations, $20 \mathrm{ml}$ of mCPSM were inoculated with $1 \%(\mathrm{v} / \mathrm{v})$ of the preculture and incubated during $48 \mathrm{~h}$ at $37^{\circ} \mathrm{C}$ for $\mathrm{LAB}$ and at $25^{\circ} \mathrm{C}$ for yeasts. Two technical replicate fermentations were performed per strain. Samples were collected after $48 \mathrm{~h}$ of incubation and centrifuged at $14,000 \times \mathrm{g}$ for $12 \mathrm{~min}$. The supernatants were diluted 1:5 (vol/vol) with Milli-Q water, filtered $\left(0.2 \mu \mathrm{m}\right.$, Sartorius $\mathrm{AG}$, Goettingen, Germany), and stored at $-20{ }^{\circ} \mathrm{C}$ until analysis. Residual amounts of the substrates glucose, fructose, and citric acid and the amount of produced lactic acid, acetic acid, ethanol, and mannitol were determined in the fermentation samples using high-performance liquid chromatography with refractive index detector (HPLCRI) according to Romanens et al. (2018). The acidification property of LAB was determined by measuring the $\mathrm{pH}$ in samples taken from the $\mathrm{LAB}$ cultures after $48 \mathrm{~h}$ with a $\mathrm{pH}$ meter $(\mathrm{pH}-$ Meter 761 Calimatic, Merck). 


\section{Determining growth of LAB and yeast strains under stress conditions}

Growth curves in mCPSM were recorded under optimal reference conditions of LAB at $37^{\circ} \mathrm{C}$ and of yeasts at $25{ }^{\circ} \mathrm{C}$ and under stress conditions by adding different concentrations of acetic acid, lactic acid, and/or ethanol to mCPSM or by applying increased incubation temperatures (Tab. 1). The weights to calculate the weighted average growth ( $\left.\varnothing_{\text {Growth }}\right)$ were defined according to the importance of the stress factors during cocoa bean fermentation. Growth in presence of single metabolites was weighted with $30 \%$, i.e. with $10 \%$ for each single metabolite, in presence of combined metabolites with $35 \%$, and at elevated temperatures with $35 \%$ and subdivisions were made where necessary. After addition of the chemicals, which were purchased from Sigma-Aldrich (Saint Louis, USA), to mCPSM, the medium's pH was readjusted to 4.0 with $10 \mathrm{M} \mathrm{NaOH}$ solution.

Table 1. Stress conditions applied to screen LAB and yeast strains for stress tolerance including weights per

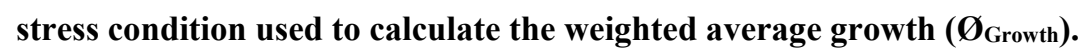

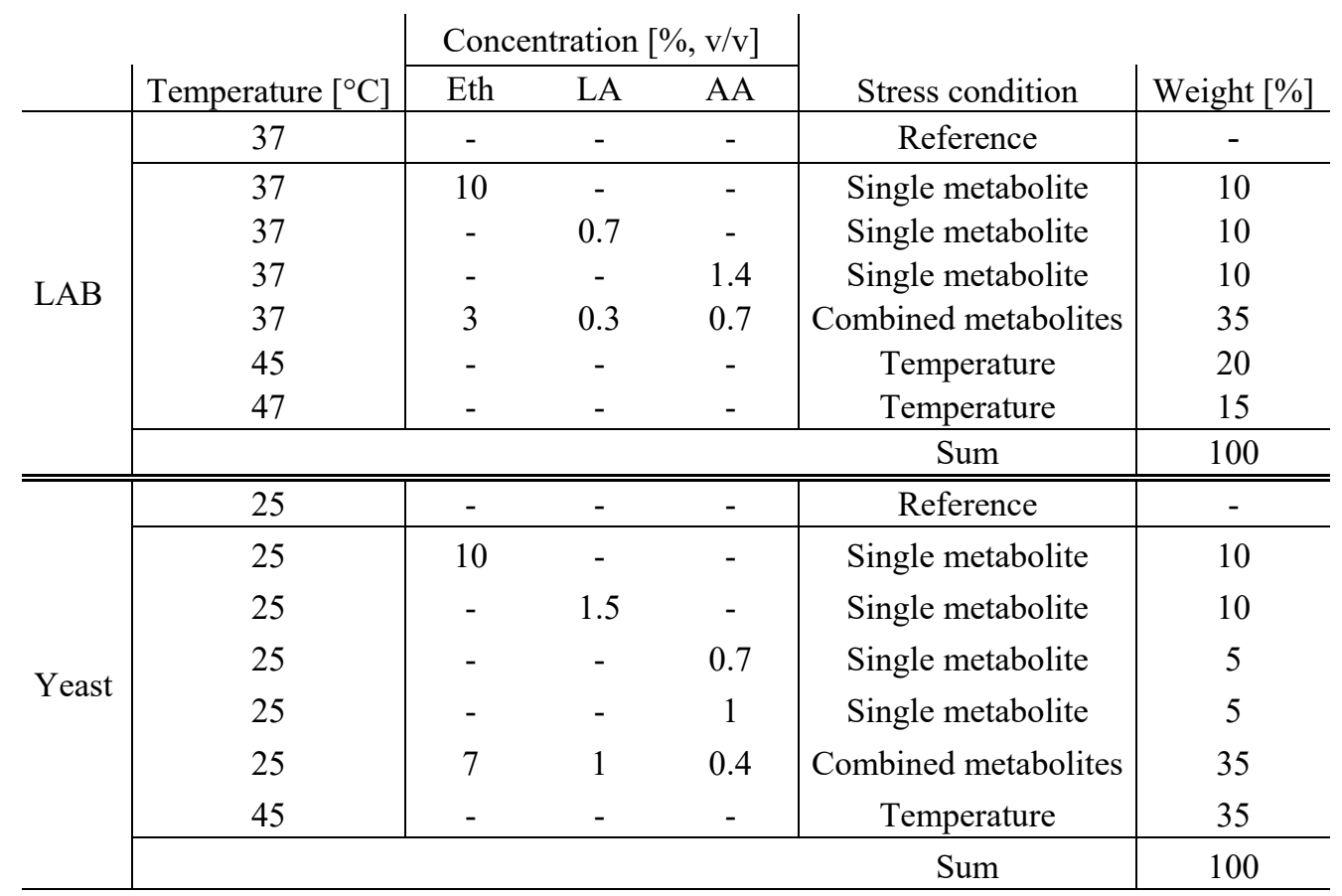

To calculate the $\emptyset_{\text {Growth }}$ for LAB and yeasts, growth in presence of single metabolites was weighted with $30 \%$, in presence of combined metabolites with $35 \%$, and at elevated temperatures with $35 \%$ of total weight. Eth = ethanol; LA = lactic acid; AA = acetic acid; - = not applicable

For the precultures, mCPSM was inoculated with pure colonies from mCPSM agar plates and incubated for $5-7 \mathrm{~h}$ at $37^{\circ} \mathrm{C}$ for $\mathrm{LAB}$ and $17-23 \mathrm{~h}$ at $25^{\circ} \mathrm{C}$ for yeasts. After centrifugation, the pellets were resuspended in fresh mCPSM and diluted to an absorbance value of 1.0 optical 
densitiy $\left(\mathrm{OD}_{600}\right)$. One percent $(\mathrm{v} / \mathrm{v})$ thereof was inoculated in $250 \mu 1$ of the respective medium in 96-well plates, leading to an initial absorbance value of $0.01 \mathrm{OD}_{600}$. Growth was monitored by measuring $\mathrm{OD}_{600}$ after a shaking step every hour during $48 \mathrm{~h}$ in a microplate reader (Biotek Synergy 1, Vermont, US) or a Bioscreen C (Oy Growth Curves Ab Ltd, Helsinki, Finland). Per stress condition and strain combination, three biological replicate measurements were performed and growth was calculated by dividing the maximal OD 600 reached during $48 \mathrm{~h}$ of incubation under the stress condition by the maximal $\mathrm{OD}_{600}$ reached at the respective reference condition. Per LAB and yeast strain, the weighted average growth ( $\left.\varnothing_{\text {Growth }}\right)$ was calculated over all stress conditions using the weights listed in Tab. 1.

\section{Analysis of genomic sequences of LAB and yeast strains}

Genomic DNA of LAB strains was isolated using a lysozyme-based cell wall digestion step and the Wizard genomic DNA purification kit (Promega, Dübendorf, Switzerland) as described previously (Inglin et al., 2017) and was sequenced using Illumina Miseq 125-bp paired-end sequence technology. The raw reads were imported and paired in the CLC genomics workbench 8.0 and assembled using the following settings: the automatic word and bubble size option was activated and the minimum contig length set at $2000 \mathrm{bp}$. Contigs were exported as nucleotide FASTA files for further analyses. Protein-encoding sequences (CDSs) were predicted using a heuristic HMM algorithm available at Genmark (http://exon.gatech.edu/GeneMark/). Predicted CDS were compared to the protein version of the Comprehensive Antibiotic Resistance Database (McArthur et al., 2013; https://card.mcmaster.ca/about), version 1.1 .1 from $5^{\text {th }}$ October 2016 using basic alignment search tool protein (BLASTP). BLAST was locally performed using the CLC genomic workbench 8.0, using a cut off value of $\mathrm{e}<10^{-20}$. Virulence factors were identified by BLASTP with the predicted proteome against the full set of proteins from the virulence factor database (http://www.mgc.ac.cn/VFs/, downloaded on $27^{\text {th }}$ October 2016). Cut off values were identical as for antibiotic resistance identification. LAB strains were identified using a co-occurring k-mers mapping at KmerFinder 2.0 (https://cge.cbs.dtu.dk/services/KmerFinder-2.0/) (Hasman et al., 2014; Larsen et al., 2014).

Yeast strains were identified by sequencing the rRNA gene internal transcribed spacer (ITS) region. Fungal DNA was extracted using the ZR Fungal/Bacterial DNA MiniPrep Protocol (Zymo Research Corp.). The ITS region was amplified with ITS1 and ITS4 primers according to Glass and Donaldson (1995) and PCR amplicons were subjected to Sanger sequencing (GATC Biotech, Konstanz, Germany). The sequences were compared with sequences available in the NCBI 18S rRNA genes database using BLAST. 


\section{Results}

\section{Mycotoxin production by three filamentous fungal strains}

Three filamentous fungal strains, i.e. A. flavus S075, G. moniliformis S003, and P. citrinum S005, were tested for their potential to produce mycotoxins on gene level and phenotypically. PCR amplification products for genes responsible for aflatoxin biosynthesis, i.e. aflD, aflO and aflP, were achieved with $A$. flavus S075 and this strain also produced aflatoxin when tested on YCSD agar. However, the concentrations of aflatoxins B1, B2, G1 and G2 were below the limit of detection of $0.5 \mu \mathrm{g} / \mathrm{kg}$ when tested on cocoa beans. The relevant genes for fumonisin production, fuml and fum19, were found in G. moniliformis S003, but when beans were inoculated with this strain, neither fumonisin B1 nor B2 was detected at a limit of detection of $50 \mu \mathrm{g} / \mathrm{kg}$. P. citrinum S005 produced citrinin both on CCA agar and on cocoa beans, and a concentration of $61 \mathrm{mg} / \mathrm{kg}$ was measured on the latter.

\section{Inhibition of filamentous fungi in vitro}

Anti-fungal activities in vitro of LAB and yeasts as revealed by agar spot assays are demonstrated (Tab. 2 and 3) in the following section. In terms of LAB, 362 cocoa-derived strains were tested on buffered MRS against G. moniliformis S003 and P. citrinum S005 and average scores of inhibition (Ø $\left.\varnothing_{\mathrm{SI}}\right)$ are shown per LAB strain (Tab. 2). In the genera Lactobacillus and Pediococcus, 88 out of 244 and 6 out of 46 strains, respectively, were categorized with high $\varnothing_{\mathrm{SI}}$ values of 2.6-4.0, while 36 strains belonging to the genera Enterococcus, Fructobacillus, Leuconostoc, and Weissella, were classified with medium or low $\emptyset_{\text {SI }}$ of 1.1-2.5 or 0.1.-1.0, respectively.

To select strains from the $362 \mathrm{LAB}$ strains tested on buffered MRS for anti-fungal in vitro screening on mCPSM, 64 LAB strains were preliminarily tested in an agar spot assay on mCPSM. These preliminary tests revealed higher $\emptyset_{\mathrm{SI}}$ on mCPSM for Lb. fermentum and $L b$. sp. than for Lb. paraplantarum, Lb. pentosus, and Lb. plantarum strains (data not shown). Therefore, $26 \mathrm{LAB}$ strains, i.e. strains that reached $\emptyset_{\mathrm{SI}} \geq 2.0$ on buffered MRS and had been identified as $L b$. fermentum or $L b$. sp. and strains that reached $\emptyset_{\mathrm{SI}} \geq 2.0$ on mCPSM in the preliminary tests, were selected for in vitro anti-fungal activity screening on mCPSM against seven filamentous fungal strains (Supplementary Tab. S1). All 26 LAB strains inhibited the seven tested filamentous fungal strains, i.e. displayed $\emptyset_{\mathrm{SI}}$ of at least 1.0 for each individual fungal strain, and reached medium to high Øsı of 2.3-3.1 (Supplementary Tab. S1). Over all 
LAB strains, A. candidus $\mathrm{S} 010$ was the most sensitive fungal strain inhibited on average with an SI of $3.1 \pm 0.4$, whereas the most resistant fungus was $A$. flavus S075 inhibited on average with an SI of $1.9 \pm 0.4$. Exemplary inhibition zones on mCPSM against $P$. citrinum S005 of LAB strains M017 (II), M018 (III), and M031 (VI) with SI of 2.5 are shown in Supplementary Fig. S1a.

Table 2. Average scores of inhibition (Ø $\left(_{\mathrm{SI}}\right)$ of $362 \mathrm{LAB}$ strains against $G$. moniliformis $\mathrm{S003}$ and $P$. citrinum S005 revealed by an in vitro agar plate assay on buffered MRS agar.

\begin{tabular}{l|ccc}
\multirow{2}{*}{ Species } & \multicolumn{3}{|c}{$\emptyset_{\mathrm{SI}}$} \\
\cline { 2 - 4 } Enterococcus sp. & $0.0-1.0$ & $1.1-2.5$ & $2.6-4.0$ \\
Enterococcus casseliflavus & 4 & 1 & - \\
Enterococcus faecalis & 4 & - & - \\
Enterococcus faecium & 1 & - & - \\
Enterococcus galinarium & 1 & 2 & - \\
Enterococcus sp. total & 1 & - & - \\
\hline Fructobacillus sp. & $\mathbf{1 1}$ & $\mathbf{3}$ & - \\
\hline Lactobacillus sp. & - & $\mathbf{1}$ & - \\
Lactobacillus amylovorus & 21 & 68 & 33 \\
Lactobacillus fermentum & 8 & 3 & - \\
Lactobacillus nagelii & 1 & 9 & - \\
Lactobacillus paraplantarum & - & 2 & - \\
Lactobacillus pentosus & - & - & 2 \\
Lactobacillus plantarum & - & 3 & 5 \\
Lactobacillus sp. total & - & 41 & 48 \\
\hline Leuconostoc sp. & $\mathbf{3 0}$ & $\mathbf{1 2 6}$ & $\mathbf{8 8}$ \\
Leuconostoc pseudomesenteroides & 15 & 2 & - \\
Leuconostoc sp. total & 1 & 1 & - \\
\hline Pediococcus sp. & $\mathbf{1 6}$ & $\mathbf{3}$ & - \\
Pediococcus acidilactici & 6 & 23 & 6 \\
Pediococcus sp. total & 3 & 8 & - \\
\hline Weissella sp. & $\mathbf{9}$ & $\mathbf{3 1}$ & $\mathbf{6}$ \\
\hline Not identified & $\mathbf{2}$ & - & - \\
\hline (11 & $\mathbf{1 1}$ & $\mathbf{1 7}$ & $\mathbf{8}$
\end{tabular}

ØSI represent mean values of SI against G. moniliformis S003 and P. citrinum S075, each determined as single replicates. $-=$ not applicable

In terms of yeasts, 384 strains were tested on MEA against G. moniliformis S003 and A. flavus S075 and average scores of inhibition (Ø $\varnothing_{\mathrm{SI}}$ ) are shown per yeast strain (Tab. 3). The yeast genus Trichosporon was the genus with the highest anti-fungal scores on MEA with $100 \%$ of strains with $\emptyset_{\mathrm{SI}} \geq 1.0$ and $43 \%$ with $\emptyset_{\mathrm{SI}} 2.0-6.0$. The genera Pichia and Saccharomyces demonstrated a moderate anti-fungal activity, with $63 \%$ and $33 \%$ of the strains, respectively, reaching $\emptyset_{\mathrm{SI}} \geq$ 1.0, while the genus Hanseniaspora showed the lowest anti-fungal activity with only $12 \%$ of strains with $\emptyset_{\mathrm{SI}} \geq 1.0$. 
Table 3. Average scores of inhibition (Ø $\left.\varnothing_{\mathrm{SI}}\right)$ of 384 yeast against G. moniliformis S003 and A. flavus $\mathrm{S075}$ revealed by an in vitro agar plate assay on MEA.

\begin{tabular}{|c|c|c|c|}
\hline \multirow[b]{2}{*}{ Species } & \multicolumn{3}{|c|}{$\varnothing_{\mathrm{SI}}$} \\
\hline & $0.0-0.5$ & $1.0-1.5$ & $2.0-6.0$ \\
\hline Candida glabrata & - & - & 2 \\
\hline Candida parapsilosis & 1 & 2 & - \\
\hline Candida sp. total & 1 & 2 & 2 \\
\hline Diutina rugosa & - & 3 & 2 \\
\hline Hanseniaspora guilliermondii & 1 & 1 & - \\
\hline Hanseniaspora opuntiae & 77 & 9 & - \\
\hline Hanseniaspora sp. & 36 & 7 & - \\
\hline Hanseniaspora sp. total & 114 & 17 & - \\
\hline Meyerozyma guilliermondii & 3 & - & - \\
\hline Meyerozyma sp. & 1 & - & 1 \\
\hline Meyerozyma sp. total & 4 & - & 1 \\
\hline Pichia kluyveri & 1 & - & - \\
\hline Pichia kudriavzevii & 33 & 87 & - \\
\hline Pichia manshurica & 12 & 9 & 1 \\
\hline Pichia sp. & 14 & 7 & - \\
\hline Pichia sp. total & 60 & 103 & 1 \\
\hline Rhodotorula glutinis & - & - & 1 \\
\hline Rhodotorula sp. & - & 1 & - \\
\hline Rhodotorula sp. total & - & 1 & 1 \\
\hline Saccharomyces cerevisiae & 5 & 3 & - \\
\hline Saccharomyces sp. & 21 & 7 & 3 \\
\hline Saccharomyces sp. total & 26 & 10 & 3 \\
\hline Schwanniomyces etchellsii & 4 & 2 & - \\
\hline Torulaspora delbruekii & 2 & - & - \\
\hline Torulaspora sp. & 4 & 5 & 3 \\
\hline Torulaspora sp. total & 6 & 5 & 3 \\
\hline Trichosporon asahii & - & 3 & 10 \\
\hline
\end{tabular}

$\emptyset_{\mathrm{SI}}$ represent mean values of SI against $G$. moniliformis $\mathrm{S003}$ and $A$. flavus $\mathrm{S075}$, each determined as single replicates. - = not applicable

From the 384 yeast strains tested on MEA, 63 strains with $\emptyset_{\mathrm{SI}} \geq 1.0$ were selected for in vitro agar spot assays on mCPSM against six filamentous fungal strains (Supplementary Tab. S2). The zones of inhibition for yeast strains were generally smaller and more turbid than for LAB strains (Supplementary Fig. S1b). Øsi varied considerably for different yeast strains, with values between 0.2 and 2.3, with highest $\varnothing_{\text {SI }}$ found for the Candida glabrata strains H30 and H29 with 2.3 and 2.2, respectively (Supplementary Tab. S2). Most Hanseniaspora and Saccharomyces strains reached medium $\emptyset_{\mathrm{SI}}$ between 1.0 and 2.0, with Hanseniaspora opuntiae $\mathrm{H} 17$ and Saccharomyces cerevisiae H290 showing $\varnothing_{\mathrm{SI}}$ of 1.8 and 1.5, respectively. Low $\emptyset_{\mathrm{SI}}$ of 0.0-0.9 were detected for most tested strains of the genera Diutina, Meyerozyma, Pichia, Schwanniomyces, Torulaspora, and Trichosporon. The share of filamentous fungal strains inhibited with SI $\geq 0.5$ varied from one out of six to six out of six for different yeast strains; 
yeast strains Hanseniaspora opuntiae H17 inhibited five out of six and Saccharomyces cerevisiae $\mathrm{H} 290$ all six fungal indicator strains.

\section{Carbon metabolism and acidification properties of anti-fungal LAB and yeast strains}

Anti-fungal strains, i.e. $26 \mathrm{LAB}$ and 45 yeast strains previously tested for in vitro anti-fungal activity on mCPSM (Supplementary Tab. S1 and S2) were characterized in regards to assimilation of mCPSM's substrates, production of metabolites, and, in the case of LAB, to acidification properties (Supplementary Tab. S3 and S4). In terms of yeasts, the genera Torulaspora and Trichosporon were excluded from this test due to safety concerns in view of their application in food fermentation.

Out of $26 \mathrm{LAB}$ strains, the $10 \mathrm{~g} / 1$ citric acid present in fresh mCPSM was completely metabolized within $48 \mathrm{~h}$ by 21 strains, including $L b$. fermentum strain M017, one strain was citrate-negative, and another four strains, amongst them Lb. fermentum 223, converted citric acid incompletely (Supplementary Tab. S3). All tested LAB strains metabolized the sugars glucose and fructose, both present at levels of $25 \mathrm{~g} / 1$ in fresh mCPSM. Fructose appeared to be converted preferentially, leading to an accumulation of mannitol at concentrations of 10.4$26.4 \mathrm{~g} / \mathrm{l}$. Lactic and acetic acids were produced by all 26 tested strains, at concentrations of 6.3$11.2 \mathrm{~g} / 1$ and 3.3-7.9 g/l, respectively. Lb. fermentum strain M017 produced $8.6 \mathrm{~g} / 1$ lactic acid and $6.2 \mathrm{~g} / \mathrm{l}$ acetic acid and Lb. fermentum $22310.3 \mathrm{~g} / 1$ lactic acid and $3.9 \mathrm{~g} / \mathrm{l}$ acetic acid. The $\mathrm{pH}$ measurements showed that the medium's initial $\mathrm{pH}$ of 4.0 remained stable or was slightly lowered resulting in final $\mathrm{pH}$ values of 3.4-4.0 (Supplementary Tab. S3).

The substrate and metabolite analyses of 45 yeast strains revealed that none of the yeast strains metabolized citric acid (Supplementary Tab. S4). Both glucose and fructose were used, glucose being consumed preferentially, to produce ethanol at concentrations of up to $13.2 \mathrm{~g} / 1$ mainly by yeasts belonging to the genera Candida, Hanseniaspora, Meyerozyma, Pichia, and Saccharomyces, whereas yeasts of the genus Diutina produced no ethanol. Sacc. cerevisiae produced $7.4 \mathrm{~g} / 1$ of ethanol, whereas no ethanol was produced by H. opuntiae H17.

\section{Stress tolerance of anti-fungal LAB and yeast strains}

To investigate the physiological adaptation of anti-fungal LAB and yeast strains to the cocoa bean fermentation environment, the growth of the $26 \mathrm{LAB}$ and 45 yeast strains (section 2.4.3) was recorded in mCPSM under different stress conditions (Fig. 1; complete data sets in 
Supplementary Tab. S3 and S4). Percentage growth refers to OD 600 under stress conditions relative to $\mathrm{OD}_{600}$ measured at the respective reference condition and at growth values of $\geq 10 \%$, the strains were considered as tolerant towards the tested stress condition.

Out of 26 tested LAB strains, 24 tolerated 10\% ethanol, $260.7 \%$ lactic acid, $251.4 \%$ acetic acid, and 22 a combination of 3\% ethanol, $0.3 \%$ lactic acid, and $0.7 \%$ acetic acid (Fig. 1a; Supplementary Tab. S3). A high temperature tolerance was seen for LAB, with 24 out of 26 strains tolerating $45^{\circ} \mathrm{C}$ and four also $47^{\circ} \mathrm{C}$. The two Lb. fermentum strains M017 and 223 were tolerant towards all tested conditions except for temperatures of $47^{\circ} \mathrm{C}$ and strain $\mathrm{M} 017$ generally appeared more stress tolerant than 223 , especially towards $10 \%$ ethanol and $45^{\circ} \mathrm{C}$ resulting in weighted average growth rates $\left(\varnothing_{\text {Growth }}\right)$ of $53 \%$ for M017 and of $33 \%$ for 223 (Supplementary Tab. S3).

Amongst yeast strains, the highest tolerance towards $10 \%$ ethanol was found for the 13 tested Saccharomyces strains, showing growth rates close to $100 \%$ for 11 strains (Fig. 1c). However, seven out of nine Hanseniaspora strains (Fig. 1b), two of three Candida strains and all ten tested Pichia strains also tolerated $10 \%$ ethanol, while no ethanol tolerance was recorded for Diutina, Rhodotorula, and Schwanniomyces strains (Supplementary Tab. S4). All 45 tested yeast strains tolerated a concentration of $1.5 \%$ lactic acid, a great part of the strains with growth values around $100 \%$. Diutina and Pichia were the most resistant against acetic acid of the ten tested yeast genera, followed by Saccharomyces with all strains tolerating $0.7 \%$ acetic acid and eight out of 13 strains 1.0\% acetic acid. The genus Hanseniaspora was one of the less acetic acid tolerant yeast genera, with only five of nine strains tolerating $0.7 \%$ acetic acid. Saccharomyces and Pichia strains, as well as two of nine Hanseniaspora strains tolerated the combined metabolites, i.e. $7 \%$ ethanol, $1.0 \%$ lactic acid, and $0.4 \%$ acetic acid. For most yeasts, no growth was observed at elevated temperatures and only the two tested C. glabrata and five of ten Pichia strains grew at $45^{\circ} \mathrm{C}$ and one Pichia strain at $47^{\circ} \mathrm{C}$ (data not shown). The two yeast strains selected for future applications, H. opuntiae H17 and Sacc. cerevisiae H290 tolerated all single metabolite stress conditions except for 1.0\% acetic acid for $\mathrm{H} 17$, and $\mathrm{H} 290$ additionally tolerated the combined metabolites stress, resulting in $\emptyset_{\text {Growth }}$ of $15 \%$ for $H$. opuntiae H17 and 43\% for Sacc. cerevisiae H290, respectively (Supplementary Tab. S4). 


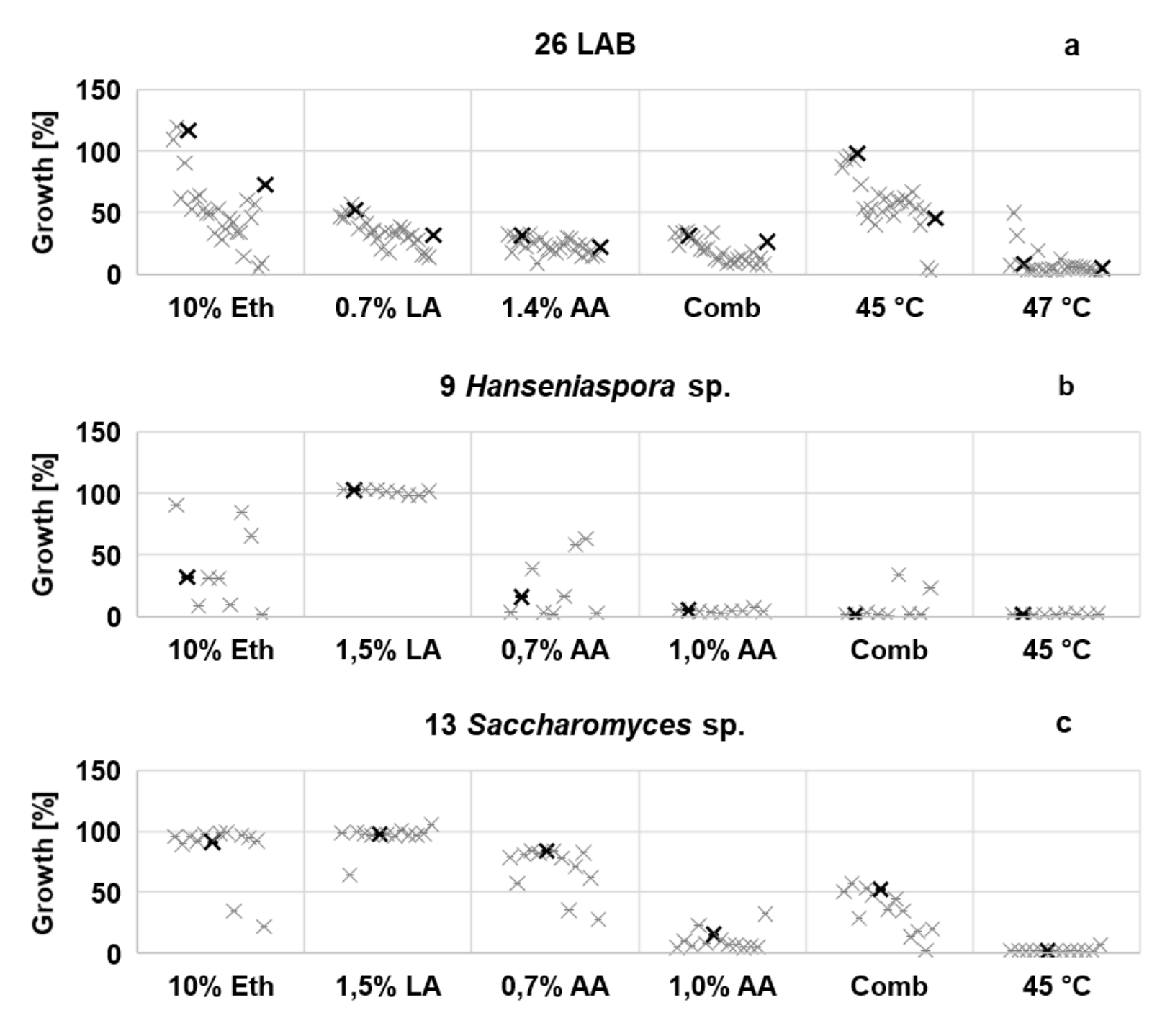

Figure 1. Growth rates determined as OD60 of 26 LAB strains (a), 9 Hanseniaspora sp. (b), and 13 Saccharomyces sp. (c) under different stress conditions relative to $\mathrm{OD}_{600}$ measured at the respective reference conditions. Different positions along the $x$-axis help to discriminate between single strains and have no further meaning. Black crosses highlight strains Lb. fermentum M017 (a; left), Lb. fermentum 223 (a; right), H. opuntiae H17 (b), and Sacc. cerevisiae $\mathrm{H} 290$ (c). Eth = ethanol; LA = lactic acid; AA = acetic acid; Comb = combination of $3 \%$ ethanol, $0.3 \%$ lactic acid, and $0.7 \%$ acetic acid (a) or $7 \%$ ethanol, $1.0 \%$ lactic acid, and $0.4 \%$ acetic acid (b and c)

\section{Inhibition of filamentous fungi in vivo}

By means of an anti-fungal assay on cocoa beans, the inhibition capacity of anti-fungal LAB and yeast strains was assessed in vivo against $A$. flavus S075, G. moniliformis S003, and $P$. citrinum S005 (Tab. 4). For this test, 14 LAB and 16 yeast strains were chosen with means of $\varnothing_{\text {Growth }}$ and normalized $\varnothing_{\text {SI }}$ on $\mathrm{mCPSM} \geq 47 \%$ for LAB (Supplementary Tab. S3) and $\geq 23 \%$ for yeasts (Supplementary Tab. S4). LAB strain 204 was excluded as it did not tolerate 1.4\% acetic acid and yeasts of the genera Candida and Pichia were excluded due to safety concerns 
in view of future usage in food applications. Examples of anti-fungal assays on cocoa beans are depicted in Fig. 2, showing cocoa beans that had been inoculated with spores of A.flavus S075, G. moniliformis S003, or P. citrinum S005 and cells of the anti-fungal strains Sacc. sp. H291 or Lb. fermentum M017 or the non-anti-fungal control strains Lb. fermentum 193 or Schwanniomyces etchellsii $\mathrm{H} 12$, respectively. In this example, filamentous fungal growth was inhibited at $100 \%$ by the anti-fungal strains and by $0 \%$ by the non-anti-fungal control strains. The anti-fungal assays on cocoa beans for LAB and yeasts revealed $98-100 \%$ of growth inhibition of A. flavus S075 for 6 LAB and 13 yeast strains after 4 days (Tab. 4). Until the end of the test duration, i.e. 10-11 days for LAB and 14 days for yeasts, beans had turned partly mouldy, resulting in $75-100 \%$ of growth inhibition for the 6 LAB strains and $0-95 \%$ for the yeast strains. Among these 6 LAB strains, 5 also inhibited growth of G. moniliformis S003 and P. citrinum S005 at 100\% during 10-11 days. Lb. fermentum strains M017 and 223 were selected based on a complete inhibition of G. moniliformis S003 and P. citrinum S005 and 88$100 \%$ growth inhibition of $A$. flavus S075 after 10-11 days and high $\emptyset_{\text {Growth of }} \geq 33 \%$ (Supplementary Tab. S3). H. opuntiae H17 was selected for its high growth inhibition of $A$. flavus S075 of $95 \%$ after 14 days and its tolerance towards single metabolites and Sacc. cerevisiae $\mathrm{H} 290$ was chosen for combining a high degree of growth inhibition of A. flavus S075 of $51 \%$ after 14 days with a tolerance towards single and combined metabolites (Supplementary Tab. S4). These selected LAB and yeast strains were combined to four LAB-yeast co-cultures, which completely suppressed growth of $A$. flavus S075 on the bean surface during 14 days. No inhibition was seen for four and incomplete inhibition for one co-culture, in which the yeast and/or the LAB strain was a non-anti-fungal control strain. Cell concentrations on the bean surface after 4 days of incubation were 9-10 $\log$ CFU/g for LAB and 5-9 $\log$ CFU/g for yeasts in anti-fungal single and co-cultures and the $\mathrm{pH}$ changes from day 0 to 4 ranged from -0.3 to +1.7 (Tab. 4). 


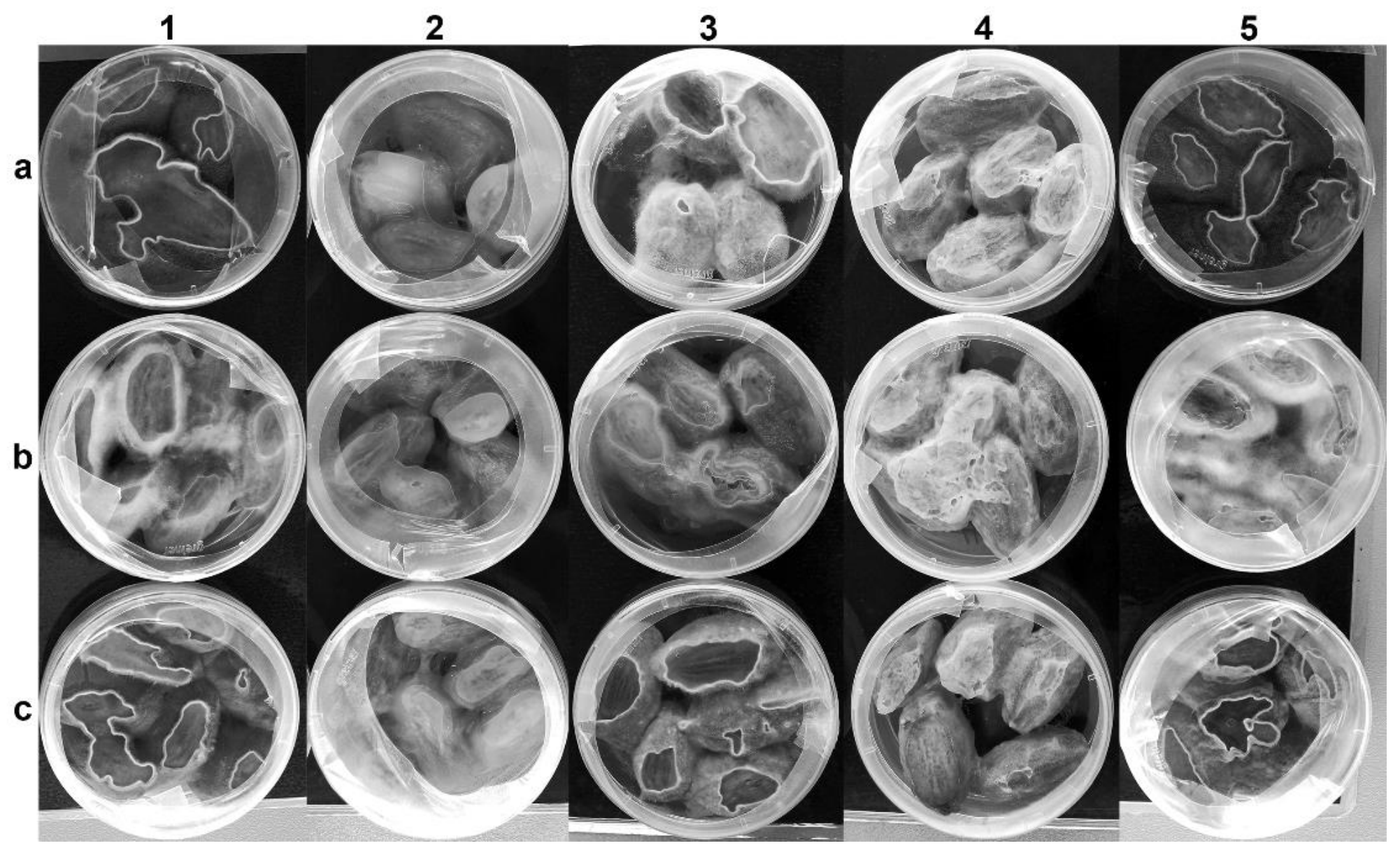

Figure 2. In vivo anti-fungal cocoa bean assay to assess growth inhibition capacity of anti-fungal LAB and yeast strains against fungal indicator strains $A$. flavus $\mathbf{S 0 7 5}$ (a), G. moniliformis S003 (b), and P. citrinum S005 (c). Column 1 shows the control with only filamentous fungi inoculated. Beans in columns 2, 3, 4, and 5 were additionally inoculated with anti-fungal $\mathbf{L b}$. fermentum M017, non-anti-fungal Lb. fermentum 193, anti-fungal Sacc. cerevisiae H290, and non-anti-fungal Sch. etchellsii H12, respectively. For better visibility of filamentous fungal growth, the contrast of the picture was increased. Filamentous fungal growth of $100 \%$ by all three indicator strains is visible in columns 1, 3, and 5, whereas in columns 2 and 4, the anti-fungal strains Lb. fermentum M017 and Sacc. cerevisiae H290, respectively, suppressed the indicator strains resulting in $0 \%$ fungal growth, i.e. $100 \%$ growth inhibition. A whitish appearance of the beans in column 4 was caused by the growth of Sacc. cerevisiae $\mathrm{H} 290$. 
Table 4. Growth inhibition of $A$. flavus $\mathrm{S075}$, pulp $\mathrm{pH}$, and cell counts as revealed by in vivo anti-fungal assay on cocoa beans for $14 \mathrm{LAB}, 16$ yeasts, and $4 \mathrm{LAB}$-yeast co-cultures.

\begin{tabular}{|c|c|c|c|c|c|c|}
\hline \multirow{3}{*}{\multicolumn{2}{|c|}{ Single- and co-cultures (no. of biological replicates) }} & \multirow{2}{*}{\multicolumn{2}{|c|}{$\begin{array}{c}\text { Growth inhibition of } \\
\text { A. flavus S075 [\%] }\end{array}$}} & \multirow{3}{*}{\begin{tabular}{|c|} 
Pulp pH \\
$\Delta$ \\
\end{tabular}} & \multicolumn{2}{|c|}{ Cell count $[\log \mathrm{CFU} / \mathrm{g}]$} \\
\hline & & & & & \multirow[t]{2}{*}{ MRS } & \multirow{2}{*}{ YGC } \\
\hline & & $\mathrm{t}_{4}$ & $t_{\text {end }}$ & & & \\
\hline \multicolumn{7}{|c|}{$\underline{\text { Lactic acid bacteria }}$} \\
\hline M038 & LAB (1) & 100 & 100 & $+0,8$ & n.d. & - \\
\hline M089* & Lb. fermentum (2) & 100 & 96 & -0.3 & 9 & - \\
\hline $222 *$ & Lb. fermentum (2) & 100 & 92 & -0.2 & 9 & - \\
\hline $223 *$ & Lb. fermentum (2) & 100 & 90 & 0.0 & 9 & - \\
\hline $\mathrm{M} 031 *$ & Lb. fermentum (2) & 100 & 75 & -0.1 & 9 & - \\
\hline M017* & Lb. fermentum (2) & 98 & 88 & -0.1 & 9 & - \\
\hline M080* & Lb. fermentum (2) & 96 & 45 & $+0,1$ & 9 & - \\
\hline M091* & Lb. fermentum (2) & 90 & 51 & $+0,1$ & 9 & - \\
\hline $221 *$ & Lb. sp. (1) & 80 & 10 & -0.1 & 9 & - \\
\hline M117B* & Lb. fermentum (1) & 40 & 0 & $+0,1$ & 9 & - \\
\hline $18 *$ & $L b$. sp. (1) & 25 & 0 & -0.1 & 9 & - \\
\hline $1 *$ & Lb. sp. (1) & 20 & 10 & 0.0 & 9 & - \\
\hline 24 & $L b$. sp. (1) & 0 & 0 & $+0,6$ & 9 & - \\
\hline M083 & $L b$. sp. (1) & 0 & 0 & -0.3 & 9 & - \\
\hline $193^{\mathrm{a}}$ & Lb. fermentum (4) & 0 & 0 & 0.0 & 9 & - \\
\hline \multicolumn{7}{|l|}{$\underline{\text { Yeasts }}$} \\
\hline H17 & H. opuntiae (3) & 100 & 95 & $+0,4$ & - & 6 \\
\hline H362 & Sacc. sp. (3) & 100 & 91 & $+0,4$ & - & 8 \\
\hline H369 & H. sp. (3) & 100 & 88 & $+0,4$ & - & 8 \\
\hline H358 & Sacc. sp. (3) & 100 & 79 & 0.0 & - & 8 \\
\hline H361 & Sacc. sp. (3) & 100 & 67 & $+0,2$ & - & 8 \\
\hline H290 & Sacc. cerevisiae (3) & 100 & 51 & $+0,2$ & - & 8 \\
\hline $\mathrm{H} 156$ & Sacc. cerevisiae (3) & 100 & 45 & $+0,4$ & - & 8 \\
\hline H356 & Sacc. sp. (3) & 100 & 36 & $+0,1$ & - & 8 \\
\hline H357 & Sacc. sp. (3) & 100 & 33 & $+0,1$ & - & 8 \\
\hline H311 & H. sp. (3) & 100 & 33 & $+0,1$ & - & 8 \\
\hline H363 & Sacc. sp. (3) & 100 & 13 & $+0,3$ & - & 8 \\
\hline H291 & Sacc. sp. (3) & 100 & 7 & $+0,1$ & - & 9 \\
\hline H323 & H. sp. (2) & 100 & 0 & $+0,4$ & - & 8 \\
\hline $\mathrm{H} 26$ & Sacc. cerevisiae (3) & 78 & 68 & $+0,1$ & - & 8 \\
\hline $\mathrm{H} 24$ & Sacc. cerevisiae (3) & 67 & 48 & $+0,4$ & - & 8 \\
\hline H359 & Sacc. sp. (3) & 67 & 19 & $+0,1$ & - & 8 \\
\hline $\mathrm{H} 12^{\mathrm{a}}$ & Sch. etchellsii (3) & 0 & 0 & $+0,3$ & - & 8 \\
\hline
\end{tabular}

LAB counts on MRS, yeast counts on YGC agar, and pulp pH were determined on beans inoculated only with LAB or yeast strains, respectively. Strains highlighted in bold were finally selected for future applications. * LAB strains inhibited growth of $G$. moniliformis $\mathrm{S} 003$ and $P$. citrinum $\mathrm{S} 005$ at $100 \%$ after 10-11 days (data not available for yeasts); $t_{4}=$ day $4 ; t_{\text {end }}=$ day 10-11 for LAB and day 14 for yeasts; $\Delta=$ difference in pulp pH between day 4 and day $0 ;-=$ not applicable; n.d. = not determined; $<=$ below detection limit of $2 \log \mathrm{CFU} / \mathrm{g}$; ${ }^{a}$ non-anti-fungal control strains; ${ }^{\mathrm{b}} 1$ out of 9 values above detection limit with 2 log $\mathrm{CFU} / \mathrm{g}$ 
Table 4. continued.

\begin{tabular}{|c|c|c|c|c|c|}
\hline \multirow[b]{2}{*}{ Single- and co-cultures (no. of biological replicates) } & \multicolumn{2}{|c|}{$\begin{array}{l}\text { Growth inhibition of } \\
\text { A. flavus S075 [\%] }\end{array}$} & \multirow{2}{*}{$\begin{array}{c}\text { Pulp pH } \\
\Delta \\
\end{array}$} & $\begin{array}{c}\text { Cell cou } \\
\text { MRS } \\
\end{array}$ & $\begin{array}{l}\mathrm{CFU} / \mathrm{g}] \\
\mathrm{YGC}\end{array}$ \\
\hline & $\mathrm{t}_{4}$ & $t_{\text {end }}$ & & \multicolumn{2}{|c|}{$\mathrm{t}_{4}$} \\
\hline \multicolumn{6}{|l|}{ LAB-yeast co-cultures } \\
\hline Lb. fermentum 223 x H. opuntiae H17 (1) & 100 & 100 & +0.5 & 10 & 6 \\
\hline Lb. fermentum 223 x Sacc. cerevisiae $\mathrm{H} 290$ (1) & 100 & 100 & -0.1 & 9 & 8 \\
\hline Lb. fermentum 223 x Sch. etchellsii $\mathrm{H}_{12}{ }^{\mathrm{a}}$ (1) & 0 & 0 & -0.2 & 10 & 5 \\
\hline Lb. fermentum M017 x H. opuntiae H17 (1) & 100 & 100 & +0.1 & 9 & 5 \\
\hline Lb. fermentum M017 x Sacc. cerevisiae H290 (1) & 100 & 100 & +0.1 & 9 & 8 \\
\hline Lb. fermentum M017 x Sch. etchellsii H12a (1) & 70 & 0 & +0.3 & 9 & 4 \\
\hline Lb. fermentum $193^{\mathrm{a}} \mathrm{x}$ H. opuntiae $\mathrm{H} 17$ (1) & 0 & 0 & 0.0 & 9 & $<$ \\
\hline Lb. fermentum $193^{\text {a }}$ x Sacc. cerevisiae H290 (1) & 0 & 0 & +1.7 & 9 & 8 \\
\hline Lb. fermentum $193^{\mathrm{a}}$ x Sch. etchellsii $\mathrm{H} 12$ (1) & 0 & 0 & +0.8 & 9 & 8 \\
\hline \multicolumn{6}{|l|}{ Controls } \\
\hline A. flavus $\mathrm{S} 075$ & 0 & 0 & - & - & - \\
\hline No cultures (24) & - & - & $+0,2$ & 4 & $<b$ \\
\hline
\end{tabular}

\section{Identity and safety of anti-fungal LAB and yeast strains}

LAB and yeast strains providing anti-fungal potential for future applications were identified by means of molecular biological methods. Anti-fungal LAB strains M017, M031, M080, M089, M091, 222, 223 and the non-anti-fungal control strain 193 were identified through genome sequencing as $L b$. fermentum (Supplementary Tab. S1). By sequencing the ITS region, the yeast strains H290 and H17 were identified as Sacc. cerevisiae and H. opuntiae, respectively (Supplementary Tab. S2). To assess safety criteria of Lb. fermentum strains M017 and 223, their genomes were tested for known antibiotic resistance and virulence genes. These revealed, for both strains, a hit for chloramphenicol resistance gene catB10 from Pseudomonas aeruginosa with sequence identity of amino acids of $40 \%$ and $42 \%$, respectively, but no virulence genes. The resistance could not be confirmed phenotypically in a disc diffusion test. Therefore, Lb. fermentum strains M017 and 223 can be considered as safe according to criteria applied by the European Food Safety Authority (EFSA) (EFSA, 2013; Ricci et al., 2017).

\section{Discussion}

Several studies have suggested that screening on strain level is necessary to select suitable culture strains for the cocoa bean fermentation due to large intraspecies differences (Pereira et al., 2017, 2016). In the current study, a multiphasic approach was applied to select well-adapted 
anti-fungal LAB and yeast strains for the cocoa bean fermentation from a large number of LAB and yeast strains derived from cocoa bean post-harvest processes.

An important part of this study was the screening for anti-fungal activity, in which LAB and yeasts were tested against up to seven filamentous fungal species that reflect the fungal community of the Honduran cocoa bean fermentation (Freimüller Leischtfeld, unpublished data). The biosynthesis of aflatoxin and citrinin was demonstrated for A. flavus S075 and $P$. citrinum S005 on agar plates and cocoa beans, respectively, and genes involved in fumonisin production were detected in the genome of G. moniliformis S003.

As a first step in the anti-fungal screening process of LAB and yeast strains, an agar spot assay was applied as a high-throughput method to preselect strains with anti-fungal activity. For this test, two different growth media were used, which greatly affected the resulting anti-fungal scores in the case of LAB and to a lesser extent for yeasts. Lb. plantarum, as well as Lb. pentosus and $L b$. paraplantarum, which are closely related to $L b$. plantarum according to Stiles and Holzapfel (1997), showed high scores of inhibition on buffered MRS but not on mCPSM agar, whereas $L b$. fermentum was the main anti-fungal LAB species on mCPSM. A possible reason for this might be the different metabolisms of the two LAB species, as Lb. fermentum strains produce more acetic acid and Lb. plantarum more lactic acid (Adler et al., 2013; Lefeber et al., 2011b). The difference in $\mathrm{pH}$ of the growth media, 6.4 in buffered MRS and 4.5 in mCPSM, might have affected the inhibitory effect of these acids as described by Copetti et al. (2012); or as mentioned in the review of Dalié et al. (2010), $\mathrm{pH}$ and/or nutrient composition of the media could have influenced the production of anti-fungal substances. It is assumed that MCPSM is more suitable to select antifungal LAB and yeast strains for the cocoa bean fermentation, as the $\mathrm{pH}$ and the composition of mCPSM are similar to the ones of cocoa pulp in contrast to MRS with a higher $\mathrm{pH}$ and a different nutrient composition. Finally, the screening on mCPSM agar against seven filamentous fungal species, five Aspergillus spp., one Penicillium spp., and one Gibberella spp., revealed broadband anti-fungal activity for the tested LAB and yeast strains, indicating that the anti-fungal strains used in the present study might also inhibit other filamentous fungi, e.g. OTA-producing strains.

In a final step, a cocoa bean assay specifically designed for this study was applied to screen LAB and yeast single- and co-cultures at smallest scale using $20 \mathrm{~g}$ of cocoa-pulp bean mass. The anti-fungal activity observed in vitro on mCPSM was confirmed in vivo for $L b$. fermentum, Saccharomyces, and Hanseniaspora strains with 100\% growth inhibition of A. flavus S075 on the bean surface for 4 days and for $L b$. fermentum strains that completely prevented growth of 
P. citrinum S005 and G. moniliformis S003 during 14 days. A. flavus S075 showed a high inhibition resistance against LAB strains in this test and in vitro on $\mathrm{mCPSM}$ and was therefore used as indicator strain to assess the anti-fungal activity of LAB-yeast co-cultures. By combining anti-fungal LAB and yeast strains into co-cultures, complete growth inhibition of $A$. flavus S075 was prolonged to 14 days, compared to 4 days for LAB and yeast single cultures, indicating an interaction of LAB and yeast strains in anti-fungal activity mechanisms.

In terms of anti-fungal mechanisms, there was no evidence that a change in $\mathrm{pH}$ was responsible for the anti-fungal activity of LAB strains, indicating that other mechanisms are involved in the suppression of filamentous fungi, such as the secretion of anti-fungal compounds, e.g. organic acids, cyclic dipeptides, fatty acids, or proteinaceous compounds (Brosnan et al., 2014; Crowley et al., 2013; Miescher Schwenninger et al., 2008). Surprising were the low concentrations of 2 and $3 \log \mathrm{CFU} / \mathrm{g}$ of yeast cells in single and co-culture tests that sufficed to inactivate $6 \log$ $\mathrm{CFU} / \mathrm{g}$ filamentous fungal spores, compared to $6 \log \mathrm{CFU} / \mathrm{g}$ of inoculate in the case of LAB. It indicates that the yeasts used different mechanisms than the LAB to prevent filamentous fungal growth, such as in the competition for limiting nutrients and space or production of lytic enzymes (Spadaro and Droby, 2016). Anti-fungal mechanisms responsible for the inhibitory activity of the LAB and yeast strains remain known.

To assess the anti-fungal LAB and yeast strains' fermentative potential, concentrations of main substrates and metabolites were determined after fermentation in mCPSM. The breakdown of citric acid that was observed for most tested LAB strains, is ascribed to the use of citric acid as a co-substrate in heterolactate fermentation by citrate-positive LAB species, as described by De Vuyst and Weckx (2016), and is an important role of LAB in the cocoa bean fermentation (H. D. Ouattara et al., 2017). Most tested LAB strains were more efficient in converting citric acid than sugars similarly to the findings of Pereira et al. (2012). The tested LAB species consumed both glucose and fructose, fructose being consumed almost completely and converted mostly into mannitol, as described previously by Adler et al. (2013). The tested LAB strains, i.e. 24 Lb. fermentum and 2 not further identified LAB strains, produced lactic and acetic acid. The final concentrations of 6-11 g/l and 3-8 g/l were in a similar range to the amounts of 6-13 $\mathrm{g} / \mathrm{l}$ and 5-11 g/l of lactic and acetic acid, respectively, that were measured for cocoa-derived $L b$. fermentum strains under comparable conditions (Adler et al., 2013; Lefeber et al., 2011b, 2010; Pereira et al., 2012). A moderate lactic acid production is an important characteristic for potential culture strains, as excessive amounts of the non-volatile acid can negatively affect the fermented beans acidity according to Lefeber et al. (2010). In contrast, acetic acid has been described as being important as its diffusion into the beans, together with ethanol and heat, is 
responsible for the death of the embryo and drives biochemical conversions inside the cotyledon (De Vuyst and Weckx, 2016; Ozturk and Young, 2017).

All tested yeast strains were citrate negative, which is in accordance with the reports of Fernández Maura et al. (2016) and Pereira et al. (2012), who did not find citrate conversion amongst various tested cocoa-derived yeast strains. Conversely, citrate conversion has been described for cocoa-derived yeast strains, $P$. kudriavzevii and Candida sp. being the most often mentioned yeast species with this characteristic (Daniel et al., 2009; Samagaci et al., 2016). The majority of the yeast strains converted the sugars glucose and fructose simultaneously, glucose being used in slightly higher amounts than fructose, which has been described previously by Pereira et al. (2012). The reason for poor or non-existent ethanol production of some tested yeast strains might be the lack of fresh medium caused by not shaking the fermentation tubes. Higher ethanol levels were observed, when some of the strains were fermented in continuously shaken tubes, e.g. $18.4 \mathrm{~g} / \mathrm{l}$ for the strain Hanseniaspora opuntiae H17 (data not shown). A tendency for higher ethanol production was observed for yeasts with higher $\emptyset_{\mathrm{SI}}$ on mCPSM agar and higher $\emptyset_{\text {Growth }}$ in mCPSM, i.e. strains of the genera Candida, Hanseniaspora, Pichia, and Saccharomyces, which might be ascribed to a well-adapted metabolism of these yeasts to mCPSM.

Concurrently, anti-fungal LAB and yeast strains were screened for their physiological adaptation to the cocoa bean fermentation environment by measuring growth in mCPSM under different stress conditions. For that, conditions commonly occurring during the fermentation of cocoa beans were chosen, such as high ethanol and acid levels and elevated temperatures. At the time point of maximal LAB counts, up to $1-6 \%$ ethanol, $0.1-1.7 \%$ lactic acid, $0.1-2.0 \%$ acetic acid, and temperatures of up to $35-48^{\circ} \mathrm{C}$ have been reported in the cocoa pulp bean mass (Camu et al., 2007; Lagunes Gálvez et al., 2007; Nielsen et al., 2007; Papalexandratou et al., 2013, 2011c; Romanens et al., 2018). LAB strains tested in the present study were highly tolerant towards ethanol and heat and to a lesser extent towards acetic acid, with a majority of strains tolerating $10 \%$ ethanol, $1.4 \%$ acetic acid, and $45^{\circ} \mathrm{C}$ and some even $47^{\circ} \mathrm{C}$. This might explain why LAB persist until the end of the fermentation process, when temperatures and acetic acid concentrations increase (De Vuyst and Weckx, 2016). The LAB strains' lactic acid tolerance was rather low with a concentration of $0.7 \%$ lactic acid reducing growth by more than $50 \%$ on average. A low lactic acid tolerance could be a main limiting factor for LAB growth in the fermentation process and is in accordance with Visintin et al. (2016), who reported lactic acid to be stressful towards cocoa-derived LAB strains. 
When yeast cell concentrations were at their maximum during spontaneous cocoa bean fermentations, metabolite levels of up to $1-6 \%$ ethanol, $0.1-1.5 \%$ lactic acid, and $0-0.4 \%$ acetic acid, and maximal temperatures of $28-30{ }^{\circ} \mathrm{C}$ have been measured (Camu et al., 2007; Lagunes Gálvez et al., 2007; Nielsen et al., 2007; Papalexandratou et al., 2013, 2011c; Romanens et al., 2018). Similarly to LAB, yeast strains showed a high ethanol tolerance, with most strains growing at $10 \%$ ethanol. The genera Saccharomyces and Pichia showed the highest growth rates, coinciding with Daniel et al. (2009), De Vuyst and Weckx (2016), Fernández Maura et al. (2016), and Pereira et al. (2012), who ascribed ethanol tolerance to cocoa-derived Sacc. cerevisiae and P. kudriavzevii strains. In contrast to LAB, yeast showed a high tolerance towards lactic acid, with all tested strains growing at 1.5\% lactic acid and the growth of Pichia strains even seeming to be favoured by lactic acid, as more than half of the tested Pichia strains grew better in the presence of lactic acid. The tolerance towards acetic acid and elevated temperatures, however, was low amongst yeasts, which, as stated by Daniel et al. (2009), explains the disappearance of yeast populations when temperature and acetic acid increase at the end of the cocoa bean fermentation. Despite the generally low acetic acid and heat tolerance of cocoa-derived yeast strains, the tolerance of several yeast strains towards $0.7 \%$ or even $1.0 \%$ of acetic acid and $45^{\circ} \mathrm{C}$ shows the potential of these strains to survive conditions occurring during the yeast phase as specified above.

Generally, the tolerance of LAB and yeast strains towards elevated concentrations of metabolites was greatly reduced when ethanol, lactic acid, and acetic acid were combined. Similar findings have been reported by Samagaci et al. (2014), who found that elevated temperature combined with ethanol, lactic acid, or acetic acid, was a main stress hindering the growth of yeast strains in the cocoa bean fermentation. In terms of yeasts, strains of the genera Pichia and Saccharomyces were best adapted to combined metabolite stress.

Based on the analysis of anti-fungal activity in vivo, carbon metabolism and stress tolerance, four anti-fungal LAB-yeast co-cultures, composed of one LAB and one yeast strain, were selected for future applications as protective cultures in the cocoa bean fermentation. The safety of the selected $L b$. fermentum strains in view of their application in a food product was approved by confirming the absence of virulence factors and functional antibiotic resistance determinants. The chloramphenicol resistance gene that was found was not functional according to the phenotypic resistance assays. In terms of yeasts, strains of H. opuntiae and Sacc. cerevisiae were selected due to the safe use of these genera in food products, as illustrated by the qualified presumption of safety (QPS) status attributed to Sacc. cerevisiae and H. uvarum by EFSA (Ricci et al., 2017). Furthermore, the selected yeast species are known to play an important role in the 
cocoa bean fermentation process (De Vuyst and Weckx, 2016; Fernández Maura et al., 2016; Papalexandratou et al., 2013). Strains of the species $P$. kudriavzevii, which were excluded due to safety aspects, might - if their safety for the cocoa-consumer can be approved - be used as protective cultures due to their high tolerance towards stress conditions. This species has been described as a good aroma producer, by Pereira et al. (2017), and as being well-adapted to the cocoa bean environment (Samagaci et al., 2016); furthermore Pichia sp. with high inhibitory effect against OTA producing filamentous fungi were reported by de Souza et al. (2017).

\section{Conclusion}

The multiphasic screening approach performed with modified cocoa pulp simulation medium was demonstrated to be a suitable method to select anti-fungal LAB-yeast cultures for the cocoa bean fermentation. Finally, four selected LAB-yeast co-cultures each composed of the $L b$. fermentum strains M017 or 223 and either S. cerevisiae H290 or H. opuntiae H17, showed $100 \%$ growth inhibition of the mycotoxin-producing strain A. flavus S075 on cocoa beans. The adaptation of the single strains to the cocoa bean fermentation, i.e. the ability of the LAB strains to metabolize citric acid and to grow under ethanol, acid, and heat stress and the yeasts ability to perform an alcoholic fermentation and to tolerate high ethanol and lactic acid levels, suggest their potential to participate in the LAB and the yeast phase of cocoa bean fermentation. Further trials should include the application of the LAB-yeast co-cultures in cocoa bean fermentations to assess their influence on the fermentation process and on the quality of the final dried beans in terms of fermentation degree and sensorial characteristics and to evaluate their efficiency in reducing the number of mouldy beans and mycotoxin concentrations.

\section{Acknowledgments}

The authors would like to thank Michael Pantic for assistance in data analysis, Alfonso Die for support with HPLC-RI analyses, the Research Group for Environmental Genomics and Systems Biology for providing the Bioscreen $\mathrm{C}$ for OD measurements, and Stella Cook for proofreading. 


\section{Supplementary material}
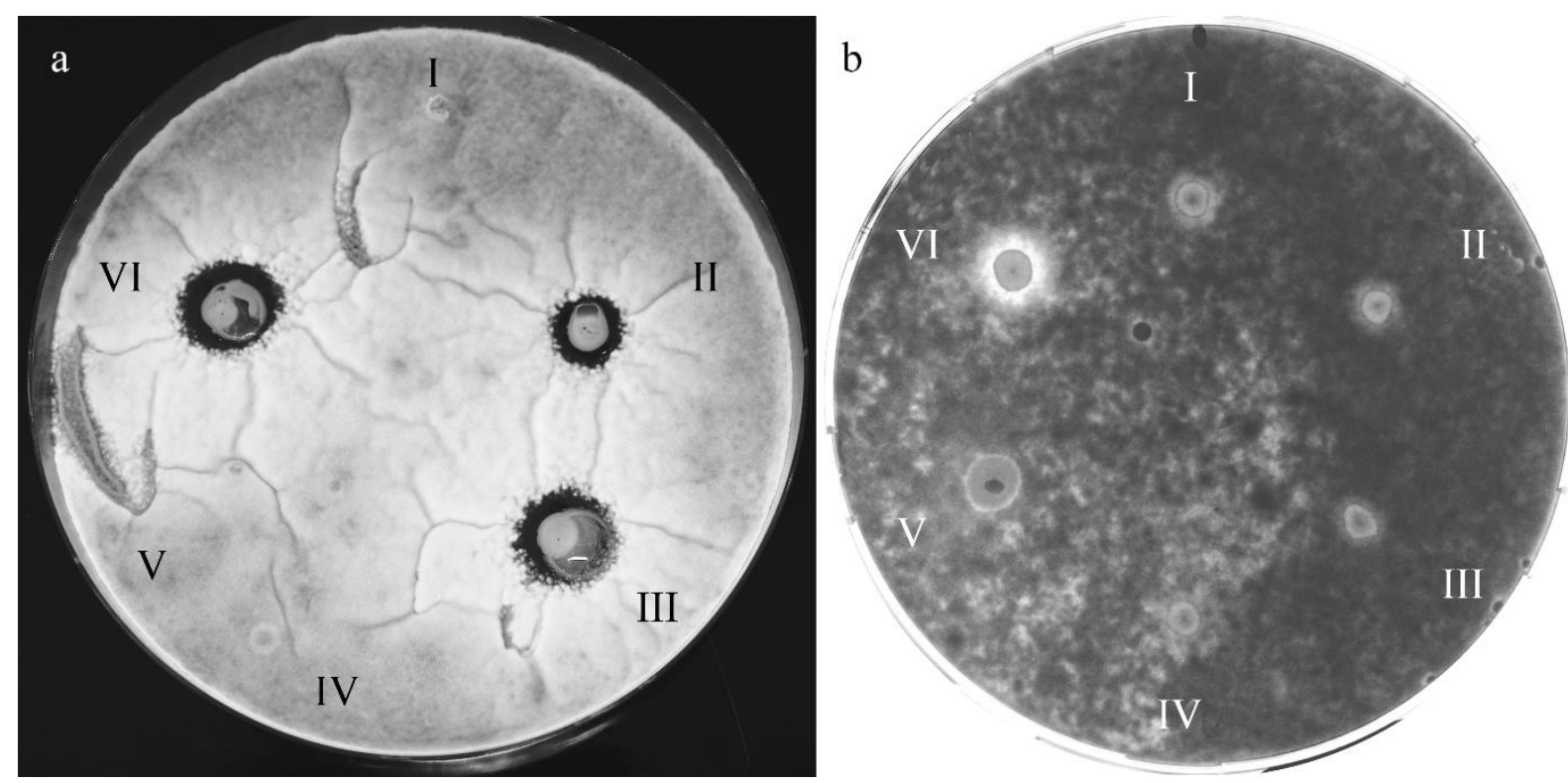

Supplementary Figure S1. Inhibition zones in an agar spot assay on mCPSM of six LAB strains against $P$. citrinum S005 (a) and six yeast strains against $A$. nidulans S049 (b) after $72 \mathrm{~h}$ of incubation at $25^{\circ} \mathrm{C}$. Inhibition of LAB in (a) was rated with a score of inhibition (SI) of 2.5 for M017 (II), M018 (III), and M031 (VI) and with SI of 0 for M016 (I), M020 (IV), and M030 (V). Yeast inhibition zones in (b) were scored with SI of 6 for H259 (VI), 1 for H258 (I), H263 (II), H262 (III), and H26 (V) and with 0 for H261 (IV). The pictures were taken on black background (a) and against light (b) and contrast was increased for better visibility of the inhibition zones. 


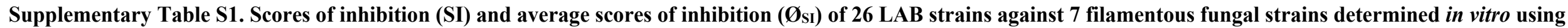
an agar plate assay on mCPSM.

\begin{tabular}{|c|c|c|c|c|c|c|c|c|c|c|}
\hline & & & & & & SI & & & & \\
\hline Test stra & & $\begin{array}{c}\text { Method of } \\
\text { identification }\end{array}$ & $\begin{array}{l}\text { A. candidus } \\
\text { S010 }\end{array}$ & $\begin{array}{l}\text { A. flavus } \\
\text { S075 }\end{array}$ & $\begin{array}{l}\text { A. nidulans } \\
\text { S049 }\end{array}$ & $\begin{array}{l}\text { A. tamarii } \\
\text { S078 }\end{array}$ & $\begin{array}{l}\text { A. versicolor } \\
\text { S085 }\end{array}$ & $\begin{array}{l}\text { G. moniliformis } \\
\text { S003 }\end{array}$ & $\begin{array}{l}\text { P. citrinum } \\
\text { S005 }\end{array}$ & $\varnothing_{\mathrm{SI}}$ \\
\hline M080 & Lb. fermentum & $\mathrm{G}$ & 3.8 & 2.5 & 3.0 & 3.3 & 3.0 & 3.5 & 3.0 & 3.1 \\
\hline 221 & $L b$. sp. & M & 3.5 & 2.5 & 2.8 & 2.8 & 3.0 & 4.0 & 3.0 & 3.1 \\
\hline 223 & Lb. fermentum & $\mathrm{G}$ & 3.5 & 2.5 & 2.5 & 2.5 & 3.5 & 3.5 & 3.0 & 3.0 \\
\hline 24 & $L b$. sp. & M & 3.5 & 2.3 & 3.5 & 1.5 & 4.0 & 3.0 & 2.8 & 2.9 \\
\hline M038 & LAB & $\mathrm{CO}$ & 3.5 & 2.0 & 2.5 & 2.8 & 3.3 & 3.5 & 3.0 & 2.9 \\
\hline 18 & $L b . \mathrm{sp}$. & $\mathrm{M}$ & 3.5 & 2.3 & 2.8 & 2.3 & 3.0 & 3.3 & 3.0 & 2.9 \\
\hline 222 & Lb. fermentum & G & 2.8 & 2.5 & 2.5 & 2.8 & 3.0 & 3.0 & 3.3 & 2.8 \\
\hline M089 & Lb. fermentum & $\mathrm{G}$ & 3.8 & 1.5 & 2.5 & 2.5 & 2.8 & 3.5 & 3.3 & 2.8 \\
\hline 44 & $L b$. sp. & M & 3.3 & 2.3 & 2.5 & 2.0 & 3.5 & 3.3 & 2.5 & 2.8 \\
\hline 26 & $L b . \mathrm{sp}$. & M & 2.8 & 2.3 & 3.0 & 1.0 & 3.5 & 3.0 & 3.3 & 2.7 \\
\hline M091 & Lb. fermentum & G & 3.3 & 1.5 & 2.5 & 2.5 & 3.3 & 2.8 & 3.0 & 2.7 \\
\hline 1 & $L b . \mathrm{sp}$ & M & 3.3 & 2.3 & 2.5 & 2.0 & 2.8 & 3.0 & 2.8 & 2.6 \\
\hline 204 & Lb. sp. & M & 3.0 & 2.0 & 2.3 & 2.0 & 2.8 & 3.5 & 2.8 & 2.6 \\
\hline M035 & $L b . \mathrm{sp}$. & M & 3.3 & 2.0 & 2.0 & 2.3 & 2.8 & 3.0 & 3.0 & 2.6 \\
\hline 19AFL & Lb. fermentum & M & 3.3 & 1.5 & 2.3 & 2.5 & 3.5 & 2.5 & 2.5 & 2.6 \\
\hline M018 & LAB & $\mathrm{CO}$ & 3.3 & 1.5 & 2.0 & 2.5 & 3.0 & 2.8 & 3.0 & 2.6 \\
\hline 38 & $L b . \mathrm{sp}$. & M & 3.0 & 2.0 & 2.8 & 1.0 & 2.8 & 3.0 & 3.3 & 2.5 \\
\hline 19B & $L b . \mathrm{sp}$. & M & 2.5 & 1.5 & 2.5 & 2.3 & 3.3 & 2.5 & 3.3 & 2.5 \\
\hline 16 & $L b$. sp. & M & 2.0 & 2.3 & 2.5 & 1.5 & 3.0 & 3.0 & 3.3 & 2.5 \\
\hline 31 & $L b . \mathrm{sp}$. & M & 2.8 & 2.0 & 2.3 & 2.0 & 3.0 & 3.0 & 2.5 & 2.5 \\
\hline 42 & $L b . \mathrm{sp}$. & M & 3.0 & 1.5 & 2.0 & 2.5 & 2.8 & 3.0 & 2.8 & 2.5 \\
\hline 19AKL & Lb. fermentum & M & 2.8 & 1.3 & 2.5 & 2.0 & 3.3 & 2.8 & 3.0 & 2.5 \\
\hline M017 & Lb. fermentum & $\mathrm{G}$ & 3.3 & 1.5 & 2.0 & 2.5 & 3.0 & 2.3 & 3.0 & 2.5 \\
\hline M083 & $L b$. sp. & M & 3.5 & 2.0 & 2.3 & 2.5 & 2.5 & 2.5 & 2.0 & 2.5 \\
\hline M031 & Lb. fermentum & $\mathrm{G}$ & 2.8 & 1.8 & 2.5 & 2.0 & 2.8 & 2.5 & 2.8 & 2.4 \\
\hline M117B & Lb. fermentum & $\mathrm{M}$ & 2.8 & 1.3 & 2.0 & 2.0 & 2.5 & 2.8 & 3.0 & 2.3 \\
\hline & & 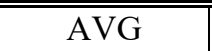 & 3.1 & 1.9 & 2.5 & 2.2 & 3.0 & 3.0 & 2.9 & \\
\hline & & STD & 0.4 & 0.4 & 0.4 & 0.5 & 0.4 & 0.4 & 0.3 & \\
\hline
\end{tabular}

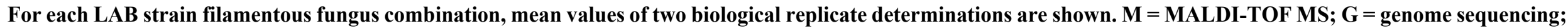
CO = catalase and oxidase tests; AVG = average STD = standard deviation 


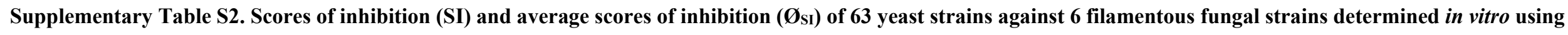
an agar plate assay on mCPSM.

\begin{tabular}{|c|c|c|c|c|c|c|c|c|c|}
\hline & & & & & & SI & & & \\
\hline Test st & rain & identification & $\begin{array}{l}\text { A. flavus } \\
\text { S075 }\end{array}$ & $\begin{array}{l}\text { A. nidulans } \\
\text { S049 }\end{array}$ & $\begin{array}{l}\text { A. tamarii } \\
\text { S078 }\end{array}$ & $\begin{array}{l}\text { A. versicolor } \\
\text { S085 }\end{array}$ & $\begin{array}{l}\text { G. moniliformis } \\
\text { S003 }\end{array}$ & $\begin{array}{l}\text { P. citrinum } \\
\text { S005 }\end{array}$ & $\emptyset_{\mathrm{SI}}$ \\
\hline H30 & C. glabrata & $\mathrm{M}$ & 1.5 & 2.0 & 1.0 & 2.0 & 1.0 & 6.0 & 2.3 \\
\hline H29 & C. glabrata & M & 1.5 & 2.0 & 0.5 & 2.0 & 1.0 & 6.0 & 2.2 \\
\hline H4 & C. parapsilosis & $\mathrm{M}$ & 1.0 & 0.0 & 0.0 & 0.0 & 0.5 & 1.0 & 0.4 \\
\hline H5 & D. rugosa & $\mathrm{M}$ & 1.5 & 0.0 & 0.5 & 0.0 & 0.0 & 0.0 & 0.3 \\
\hline H6 & D. rugosa & M & 1.5 & 0.0 & 0.5 & 0.0 & 0.0 & 0.0 & 0.3 \\
\hline H7 & D. rugosa & M & 1.5 & 0.0 & 0.0 & 0.0 & 0.0 & 0.0 & 0.3 \\
\hline H8 & D. rugosa & M & 1.5 & 0.0 & 0.0 & 0.0 & 0.0 & 0.0 & 0.3 \\
\hline H9 & D. rugosa & M & 1.5 & 0.0 & 0.0 & 0.0 & 0.0 & 0.0 & 0.3 \\
\hline H17 & H. opuntiae & $\mathrm{P}$ & 1.5 & 0.0 & 4.0 & 1.0 & 1.5 & 3.0 & 1.8 \\
\hline H311 & $H$. sp. & M & 1.5 & 0.5 & 3.0 & 2.0 & 1.5 & 1.5 & 1.7 \\
\hline H182 & H. opuntiae & M & 2.0 & 0.5 & 1.5 & 1.0 & 1.5 & 3.0 & 1.6 \\
\hline H323 & $H$. sp. & M & 3.0 & 1.0 & 1.5 & 1.5 & 0.5 & 2.0 & 1.6 \\
\hline H369 & H. sp. & M & 1.5 & 0.5 & 2.0 & 0.5 & 1.5 & 3.0 & 1.5 \\
\hline H215 & H. opuntiae & M & 1.0 & 0.0 & 2.5 & 0.5 & 1.0 & 2.0 & 1.2 \\
\hline H312 & $H$. sp. & M & 3.0 & 1.0 & 1.0 & 1.0 & 0.0 & 1.0 & 1.2 \\
\hline H186 & H. guillermondii & M & 1.0 & 0.0 & 2.0 & 0.5 & 1.0 & 2.0 & 1.1 \\
\hline H201 & H. opuntiae & $\mathrm{M}$ & 1.0 & 0.0 & 2.0 & 0.5 & 1.0 & 2.0 & 1.1 \\
\hline H289 & M. sp. & $\mathrm{M}$ & 2.0 & 1.0 & 0.0 & 1.0 & 0.0 & 0.0 & 0.7 \\
\hline H21 & P. kudriavzevii & $\mathrm{M}$ & 1.5 & 1.0 & 1.0 & 1.0 & 1.0 & 2.0 & 1.3 \\
\hline H80 & P. kudriavzevii & M & 1.5 & 0.0 & 0.5 & 0.5 & 0.0 & 1.5 & 0.7 \\
\hline H232 & P. manshurica & M & 2.5 & 0.0 & 0.0 & 0.0 & 0.0 & 1.5 & 0.7 \\
\hline H249 & P. manshurica & M & 2.5 & 0.0 & 0.0 & 0.0 & 0.0 & 1.5 & 0.7 \\
\hline H351 & $P$. sp. & M & 2.5 & 0.5 & 0.0 & 0.5 & 0.0 & 0.5 & 0.7 \\
\hline H247 & P. manshurica & M & 2.5 & 0.0 & 0.0 & 0.0 & 0.0 & 1.0 & 0.6 \\
\hline H79 & P. kudriavzevii & M & 1.5 & 0.0 & 0.0 & 0.5 & 0.0 & 1.0 & 0.5 \\
\hline H235 & P. manshurica & M & 2.5 & 0.0 & 0.0 & 0.0 & 0.0 & 0.5 & 0.5 \\
\hline H239 & P. manshurica & M & 2.5 & 0.0 & 0.0 & 0.0 & 0.0 & 0.5 & 0.5 \\
\hline
\end{tabular}

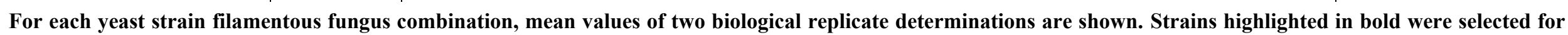

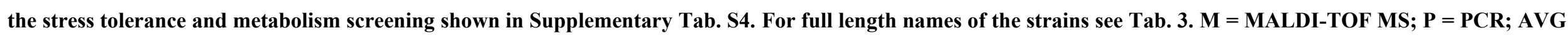
$=$ average STD = standard deviation 
Supplementary Table S2. Continued.

\begin{tabular}{|c|c|c|c|c|c|c|c|c|c|}
\hline & & & & & & SI & & & \\
\hline Test st & rain & $\begin{array}{c}\text { Method of } \\
\text { identification }\end{array}$ & $\begin{array}{l}\text { A. flavus } \\
\text { S075 }\end{array}$ & $\begin{array}{l}\text { A. nidulans } \\
\text { S049 }\end{array}$ & $\begin{array}{l}\text { A. tamarii } \\
\text { S078 }\end{array}$ & $\begin{array}{l}\text { A. versicolor } \\
\text { S085 }\end{array}$ & $\begin{array}{l}\text { G. moniliformis } \\
\text { S003 }\end{array}$ & $\begin{array}{l}\text { P. citrinum } \\
\text { S005 }\end{array}$ & $\emptyset_{\mathrm{SI}}$ \\
\hline H342 & P. sp. & $\mathrm{M}$ & 1.5 & 0.5 & 0.0 & 1.0 & 0.0 & 0.0 & 0.5 \\
\hline H250 & R. glutinis & $\mathrm{M}$ & 3.0 & 0.0 & 1.0 & 0.0 & 0.0 & 0.0 & 0.7 \\
\hline H353 & $R$. sp. & $\mathrm{M}$ & 3.0 & 0.0 & 1.0 & 0.0 & 0.0 & 0.0 & $\mathbf{0 . 7}$ \\
\hline H358 & Sacc. sp. & $\mathrm{M}$ & 1.0 & 1.5 & 2.5 & 0.5 & 2.5 & 2.5 & 1.8 \\
\hline H26 & Sacc. cerevisiae & M & 1.5 & 1.0 & 2.0 & 1.5 & 2.5 & 1.5 & 1.7 \\
\hline H362 & Sacc. sp. & M & 1.5 & 1.5 & 2.5 & 0.5 & 2.0 & 2.0 & 1.7 \\
\hline H24 & Sacc. cerevisiae & M & 1.5 & 1.0 & 2.0 & 1.5 & 2.0 & 1.5 & 1.6 \\
\hline H156 & Sacc. cerevisiae & M & 1.0 & 1.5 & 1.0 & 1.5 & 3.0 & 1.5 & 1.6 \\
\hline H363 & Sacc. sp. & M & 2.5 & 1.0 & 2.0 & 0.5 & 1.5 & 2.0 & 1.6 \\
\hline H290 & Sacc. cerevisiae & $\mathrm{P}$ & 1.0 & 1.0 & 1.5 & 1.0 & 3.0 & 1.5 & 1.5 \\
\hline H291 & Sacc. sp. & M & 1.0 & 1.0 & 1.5 & 1.0 & 3.0 & 1.5 & 1.5 \\
\hline H357 & Sacc. sp. & M & 1.0 & 1.5 & 2.5 & 0.5 & 1.0 & 2.5 & 1.5 \\
\hline H356 & Sacc. sp. & M & 1.0 & 1.0 & 1.5 & 1.5 & 2.0 & 1.5 & 1.4 \\
\hline H359 & Sacc. sp. & M & 1.0 & 1.0 & 2.5 & 0.5 & 1.5 & 2.0 & 1.4 \\
\hline H361 & Sacc. sp. & M & 1.0 & 1.5 & 1.0 & 0.5 & 2.0 & 1.5 & 1.3 \\
\hline H360 & Sacc. sp. & $\mathrm{M}$ & 1.5 & 0.0 & 0.0 & 0.0 & 0.0 & 1.0 & 0.4 \\
\hline H12 & Sch. etchellsii & $\mathrm{M}$ & 1.5 & 0.0 & 0.0 & 0.0 & 0.0 & 0.0 & 0.3 \\
\hline H15 & Sch. etchellsii & $\mathrm{M}$ & 1.5 & 0.0 & 0.0 & 0.0 & 0.0 & 0.0 & 0.3 \\
\hline $\mathrm{H} 284$ & Tor. sp. & $\mathrm{M}$ & 0.5 & 0.0 & 0.5 & 0.5 & 1.0 & 0.5 & 0.5 \\
\hline $\mathrm{H} 267$ & Tor. sp. & M & 0.5 & 0.0 & 0.0 & 0.5 & 1.0 & 0.5 & 0.4 \\
\hline $\mathrm{H} 268$ & Tor. sp. & M & 0.5 & 0.0 & 0.5 & 0.5 & 0.5 & 0.5 & 0.4 \\
\hline $\mathrm{H} 269$ & Tor. sp. & M & 0.0 & 0.0 & 0.5 & 0.5 & 1.0 & 0.5 & 0.4 \\
\hline $\mathrm{H} 285$ & Tor. sp. & M & 2.0 & 0.0 & 0.0 & 0.0 & 0.0 & 0.5 & 0.4 \\
\hline H264 & Tor. sp. & M & 0.5 & 0.0 & 0.0 & 0.5 & 0.5 & 0.5 & 0.3 \\
\hline $\mathrm{H} 265$ & Tor. sp. & M & 0.0 & 0.0 & 0.0 & 0.5 & 0.5 & 0.0 & 0.2 \\
\hline $\mathrm{H} 259$ & Trich. asahii & $\mathrm{M}$ & 0.0 & 6.0 & 0.0 & 4.0 & 0.0 & 1.0 & 1.8 \\
\hline $\mathrm{H} 254$ & Trich. asahii & M & 0.0 & 3.5 & 0.0 & 3.0 & 0.0 & 2.0 & 1.4 \\
\hline $\mathrm{H} 256$ & Trich. asahii & M & 0.0 & 2.0 & 0.0 & 1.5 & 0.0 & 0.0 & 0.6 \\
\hline $\mathrm{H} 251$ & Trich. asahii & M & 0.0 & 0.0 & 0.5 & 1.5 & 0.0 & 1.0 & 0.5 \\
\hline $\mathrm{H} 257$ & Trich. asahii & M & 0.0 & 1.0 & 0.0 & 1.5 & 0.0 & 0.5 & 0.5 \\
\hline $\mathrm{H} 255$ & Trich. asahii & M & 0.0 & 1.0 & 0.0 & 0.5 & 0.0 & 1.0 & 0.4 \\
\hline H261 & Trich. asahii & M & 0.0 & 0.5 & 0.0 & 1.0 & 0.0 & 0.5 & 0.3 \\
\hline
\end{tabular}


Supplementary Table S2. Continued.

\begin{tabular}{|c|c|c|c|c|c|c|c|c|c|}
\hline \multirow{2}{*}{\multicolumn{2}{|c|}{ Test strain }} & \multirow[b]{2}{*}{$\begin{array}{c}\text { Method of } \\
\text { identification }\end{array}$} & \multicolumn{6}{|c|}{ SI } & \multirow[b]{2}{*}{$\varnothing_{\mathrm{SI}}$} \\
\hline & & & $\begin{array}{l}\text { A. flavus } \\
\text { S075 }\end{array}$ & $\begin{array}{l}\text { A. nidulans } \\
\text { S049 }\end{array}$ & $\begin{array}{l}\text { A. tamarii } \\
\text { S078 }\end{array}$ & $\begin{array}{l}\text { A. versicolor } \\
\text { S085 }\end{array}$ & $\begin{array}{l}\text { G. moniliformis } \\
\text { S003 }\end{array}$ & $\begin{array}{l}\text { P. citrinum } \\
\text { S005 }\end{array}$ & \\
\hline $\mathrm{H} 262$ & Trich. asahii & $\mathrm{M}$ & 0.0 & 0.5 & 0.0 & 1.0 & 0.0 & 0.5 & 0.3 \\
\hline $\mathrm{H} 263$ & Trich asahii & M & 0.0 & 0.5 & 0.0 & 1.0 & 0.0 & 0.5 & 0.3 \\
\hline $\mathrm{H} 252$ & Trich. asahii & M & 0.0 & 0.0 & 0.0 & 1.5 & 0.0 & 0.0 & 0.3 \\
\hline $\mathrm{H} 258$ & Trich. asahii & M & 0.0 & 0.5 & 0.0 & 1.0 & 0.0 & 0.0 & 0.3 \\
\hline & & $\begin{array}{l}\text { AVG } \\
\text { STD }\end{array}$ & $\begin{array}{l}1.3 \\
0.9\end{array}$ & $\begin{array}{l}0.6 \\
1.0\end{array}$ & $\begin{array}{l}0.8 \\
1.0\end{array}$ & $\begin{array}{l}0.8 \\
0.8\end{array}$ & $\begin{array}{l}0.7 \\
0.9\end{array}$ & $\begin{array}{l}1.2 \\
1.2\end{array}$ & \\
\hline
\end{tabular}




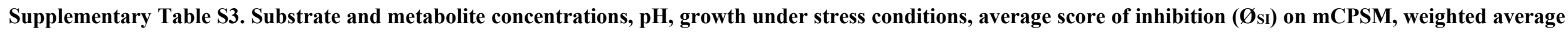
growth (Ø $\emptyset_{\text {Growth }}$ ), and mean of $\emptyset_{\text {Growth }}$ and $\emptyset_{S I}$ on mCPSM for 26 LAB strains.

\begin{tabular}{|c|c|c|c|c|c|c|c|c|c|c|c|c|c|c|c|c|c|}
\hline & \multicolumn{7}{|c|}{ Concentration $[\mathrm{g} / \mathrm{l}] / \mathrm{pH}$ at $48 \mathrm{~h}$} & \multicolumn{6}{|c|}{ Growth \pm STD [\%] } & \multirow{3}{*}{$\begin{array}{c}\varnothing_{\text {Growth }} \\
{[\%]}\end{array}$} & \multirow{3}{*}{$\begin{array}{c}\text { Normalized } \\
\emptyset_{\mathrm{SI}} \text { on } \\
\text { mCPSM [\%] }\end{array}$} & \multirow{3}{*}{$\begin{array}{c}1 / 2 \emptyset_{\text {Growth }}+1 / 2 \\
\text { normalized } \emptyset_{\mathrm{SI}} \\
\text { on mCPSM [\%] }\end{array}$} \\
\hline & & \multirow{2}{*}{ CA } & \multirow{2}{*}{ Glu } & \multirow{2}{*}{ Fru } & \multirow{2}{*}{ Man } & \multirow{2}{*}{ LA } & \multirow{2}{*}{ AA } & \multirow{2}{*}{$\mathrm{pH}$} & Eth & LA & AA & \multirow{2}{*}{ Comb $^{\mathrm{a}}$} & \multirow{2}{*}{$45^{\circ} \mathrm{C}$} & \multirow{2}{*}{$47^{\circ} \mathrm{C}$} & & & \\
\hline & & & & & & & & & $10 \%$ & $0.7 \%$ & $1.4 \%$ & & & & & & \\
\hline $\begin{array}{l}\text { Content } \\
\text { Test stra }\end{array}$ & $\begin{array}{l}n \text { mCPSM } \\
n\end{array}$ & 10.0 & 25.0 & 25.0 & 0.0 & 0.0 & 0.0 & 4.0 & - & - & - & - & - & - & - & - & - \\
\hline M080 & Lb. fermentum & $<$ & 10.7 & 1.4 & 22.8 & 8.2 & 6.6 & 3.9 & $109 \pm 28$ & $47 \pm 10$ & $32 \pm 7$ & $33 \pm 16$ & $87 \pm 1$ & $7 \pm 1$ & 50 & 79 & 64 \\
\hline M117B & Lb. fermentum & $<$ & 7.8 & 4.5 & 20.1 & 8.6 & 5.6 & 3.9 & $120 \pm 40$ & $46 \pm 40$ & $18 \pm 16$ & $23 \pm 19$ & $93 \pm 1$ & $50 \pm 5$ & 63 & 58 & 60 \\
\hline M083 & Lb. sp. & 9.4 & 10.0 & 0.3 & 24.5 & 6.3 & 3.3 & 3.9 & $62 \pm 26$ & $51 \pm 14$ & $31 \pm 10$ & $34 \pm 7$ & $96 \pm 1$ & $32 \pm 17$ & 56 & 62 & 59 \\
\hline M091 & Lb. fermentum & $<$ & 9.1 & 0.5 & 22.3 & 8.3 & 6.1 & 3.8 & $91 \pm 52$ & $57 \pm 15$ & $26 \pm 20$ & $35 \pm 11$ & $93 \pm 3$ & $8 \pm 0$ & 51 & 67 & 59 \\
\hline M017 & Lb. fermentum & $<$ & 9.0 & 0.3 & 23.0 & 8.6 & 6.2 & 3.4 & $116 \pm 44$ & $52 \pm 11$ & $31 \pm 21$ & $31 \pm 2$ & $98 \pm 1$ & $8 \pm 1$ & 53 & 63 & 58 \\
\hline М038 & LAB & $<$ & 5.2 & $<$ & 22.7 & 9.5 & 5.9 & 4.0 & $53 \pm 32$ & $37 \pm 8$ & $22 \pm 16$ & $29 \pm 10$ & $73 \pm 1$ & $3 \pm 1$ & 37 & 73 & 55 \\
\hline 24 & $L b$. sp. & $<$ & 3.3 & $0.3^{\mathrm{b}}$ & 24.0 & 10.5 & 7.2 & 3.8 & $62 \pm 14$ & $49 \pm 5$ & $33 \pm 10$ & $27 \pm 22$ & $53 \pm 1$ & $3 \pm 1$ & 36 & 73 & 54 \\
\hline 223 & Lb. fermentum & $3.9^{\mathrm{b}}$ & 11.1 & 4.5 & 20.4 & 10.3 & 3.9 & 3.9 & $73 \pm 15$ & $32 \pm 14$ & $22 \pm 16$ & $26 \pm 16$ & $45 \pm 4$ & $5 \pm 1$ & 33 & 75 & 54 \\
\hline 221 & $L b$. sp. & $<$ & 8.9 & $0.7^{\mathrm{b}}$ & 25.8 & 9.9 & 7.9 & 3.9 & $64 \pm 12$ & $41 \pm 11$ & $26 \pm 8$ & $20 \pm 10$ & $46 \pm 5$ & $4 \pm 1$ & 31 & 77 & 54 \\
\hline 204 & $L b . \mathrm{sp}$. & $4.3^{b}$ & 11.7 & 4.2 & 17.9 & 10.2 & 5.3 & 3.8 & $52 \pm 25$ & $33 \pm 22$ & $9 \pm 5$ & $19 \pm 8$ & $53 \pm 7$ & $19 \pm 2$ & 33 & 65 & 49 \\
\hline 222 & Lb. fermentum & $<$ & 8.2 & $0.7^{\mathrm{b}}$ & 26.4 & 11.2 & 5.9 & 3.9 & $50 \pm 23$ & $35 \pm 5$ & $29 \pm 27$ & $21 \pm 24$ & $40 \pm 4$ & $3 \pm 1$ & 28 & 71 & 49 \\
\hline M031 & Lb. fermentum & $<$ & 7.3 & 0.6 & 22.2 & 9.2 & 5.9 & 3.9 & $49 \pm 19$ & $29 \pm 11$ & $24 \pm 20$ & $34 \pm 23$ & $65 \pm 3$ & $2 \pm 1$ & 36 & 61 & 48 \\
\hline 18 & $L b$. sp. & $<$ & 6.9 & $<$ & 21.9 & 9.7 & 6.9 & 3.9 & $33 \pm 16$ & $21 \pm 6$ & $21 \pm 5$ & $13 \pm 9$ & $51 \pm 14$ & $4 \pm 0$ & 23 & 71 & 47 \\
\hline 1 & $L b$. sp. & $<$ & 11.2 & $0.6^{\mathrm{b}}$ & 25.6 & 10.6 & 5.7 & 4.0 & $53 \pm 20$ & $35 \pm 10$ & $20 \pm 6$ & $12 \pm 7$ & $62 \pm 7$ & $4 \pm 2$ & 29 & 66 & 47 \\
\hline M089 & Lb. fermentum & $<$ & 8.3 & 0.4 & 23.3 & 9.2 & 6.3 & 3.5 & $28 \pm 35$ & $17 \pm 5$ & $18 \pm 12$ & $17 \pm 17$ & $55 \pm 4$ & $3 \pm 2$ & 24 & 71 & 47 \\
\hline 44 & $L b . \mathrm{sp}$ & $<$ & 7.0 & 0.3 & 22.2 & 10.2 & 4.6 & 3.9 & $37 \pm 13$ & $34 \pm 19$ & $21 \pm 14$ & $8 \pm 4$ & $47 \pm 2$ & $12 \pm 14$ & 26 & 69 & 47 \\
\hline 26 & $L b . \mathrm{sp}$ & $3.5^{\mathrm{b}}$ & 10.6 & 6.4 & 16.3 & 6.8 & 5.5 & 3.8 & $45 \pm 31$ & $33 \pm 13$ & $24 \pm 16$ & $10 \pm 10$ & $59 \pm 4$ & $4 \pm 1$ & 27 & 67 & 47 \\
\hline M035 & $L b . \mathrm{sp}$ & $<$ & 7.9 & 0.9 & 21.9 & 8.6 & 6.1 & 4.0 & $42 \pm 15$ & $38 \pm 7$ & $30 \pm 4$ & $10 \pm 7$ & $59 \pm 1$ & $6 \pm 1$ & 28 & 65 & 47 \\
\hline M018 & LAB & $<$ & 7.7 & 0.7 & 23.6 & 9.5 & 6.3 & 3.9 & $34 \pm 37$ & $36 \pm 3$ & $28 \pm 6$ & $12 \pm 4$ & $62 \pm 3$ & $6 \pm 0$ & 29 & 64 & 46 \\
\hline 19B & $L b . \mathrm{sp}$. & $<$ & 8.2 & 0.5 & 22.7 & 8.5 & 6.1 & 3.9 & $34 \pm 28$ & $30 \pm 4$ & $19 \pm 7$ & $14 \pm 2$ & $58 \pm 6$ & $6 \pm 0$ & 27 & 63 & 45 \\
\hline 38 & $L b . \mathrm{sp}$ & $<$ & 9.3 & $0.6^{\mathrm{b}}$ & 21.5 & 8.8 & 6.8 & 3.9 & $14 \pm 10$ & $32 \pm 15$ & $24 \pm 13$ & $11 \pm 7$ & $67 \pm 1$ & $5 \pm 1$ & 26 & 63 & 45 \\
\hline 42 & $L b . \mathrm{sp}$ & $<$ & 8.3 & 0.4 & 23.3 & 8.6 & 6.2 & 3.8 & $60 \pm 32$ & $25 \pm 4$ & $14 \pm 6$ & $9 \pm 4$ & $54 \pm 1$ & $5 \pm 0$ & 26 & 63 & 44 \\
\hline 16 & $L b . \mathrm{sp}$. & $<$ & 8.2 & $0.6^{\mathrm{b}}$ & 23.7 & 9.8 & 7.5 & 3.9 & $46 \pm 32$ & $28 \pm 6$ & $24 \pm 4$ & $18 \pm 11$ & $40 \pm 3$ & $4 \pm 0$ & 25 & 63 & 44 \\
\hline 31 & $L b . \mathrm{sp}$ & $8.5^{\mathrm{b}}$ & 8.0 & $17.0^{\mathrm{b}}$ & 10.4 & 10.6 & $5.7^{b}$ & 3.8 & $57 \pm 33$ & $16 \pm 9$ & $17 \pm 11$ & $7 \pm 3$ & $52 \pm 6$ & $3 \pm 0$ & 23 & 63 & 43 \\
\hline 19AKL & Lb. fermentum & $<$ & 8.5 & $0.2^{\mathrm{b}}$ & 24.3 & 9.0 & 6.5 & 4.0 & $5 \pm 2$ & $16 \pm 2$ & $15 \pm 6$ & $13 \pm 10$ & $5 \pm 2$ & $2 \pm 0$ & 10 & 63 & 36 \\
\hline 19AFL & Lb. fermentum & $<$ & 10.4 & 4.6 & 19.5 & 7.6 & 5.9 & 3.9 & $9 \pm 6$ & $14 \pm 9$ & $15 \pm 3$ & $8 \pm 7$ & $2 \pm 1$ & $1 \pm 0$ & 7 & 64 & 36 \\
\hline
\end{tabular}

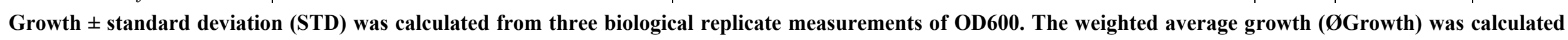

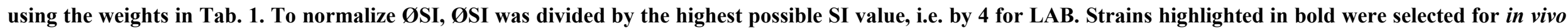

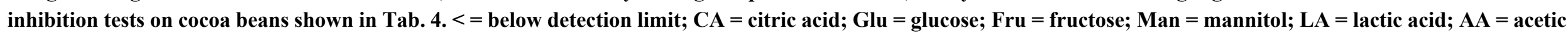
acid; Eth = ethanol; - = not applicable; a combination of $3 \%$ ethanol, $0.3 \%$ lactic acid, and $0.7 \%$ acetic acid; $b$ value below detection limit for one out of two replicates 


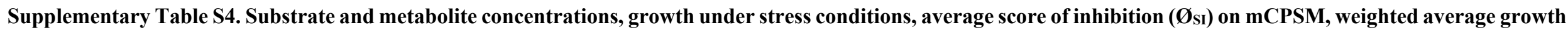
(ØGrowth), and mean of $\emptyset_{\text {Growth }}$ and $\emptyset_{\text {SI }}$ on mCPSM for 45 yeast strains.

\begin{tabular}{|c|c|c|c|c|c|c|c|c|c|c|c|c|c|c|c|c|}
\hline & \multicolumn{6}{|c|}{ Concentration $[\mathrm{g} / \mathrm{l}]$ at $48 \mathrm{~h}$} & \multicolumn{6}{|c|}{ Growth \pm STD $[\%]$} & \multirow{3}{*}{$\begin{array}{c}\emptyset_{\text {Growth }} \\
{[\%]}\end{array}$} & \multirow{3}{*}{\begin{tabular}{|c} 
Normalized \\
$\emptyset_{\mathrm{SI}}$ on \\
mCPSM [\%]
\end{tabular}} & \multirow{3}{*}{$\begin{array}{c}1 / 2 \emptyset_{\text {Growth }}+1 / 2 \\
\text { normalized } \emptyset_{\mathrm{SI}} \\
\text { on mCPSM [\%] }\end{array}$} \\
\hline & & \multirow{2}{*}{$\mathrm{CA}$} & \multirow{2}{*}{ Glu } & \multirow{2}{*}{ Fru } & \multirow{2}{*}{ LA } & \multirow{2}{*}{ AA } & \multirow{2}{*}{ Eth } & Eth & LA & \multicolumn{2}{|c|}{ AA } & \multirow{2}{*}{ Comb $^{\mathrm{a}}$} & \multirow{2}{*}{$45^{\circ} \mathrm{C}$} & & & \\
\hline & & & & & & & & $10 \%$ & $1,5 \%$ & $0,7 \%$ & $1,0 \%$ & & & & & \\
\hline \multirow{2}{*}{\multicolumn{2}{|c|}{$\begin{array}{l}\text { Content in mCPSM } \\
\text { Test strain }\end{array}$}} & 10.0 & 25.0 & 25.0 & 0.0 & 0.0 & 0.0 & - & - & - & - & - & - & - & - & - \\
\hline & & & & & & & & & & & & & & & & \\
\hline H30 & C. glabrata & 7.5 & 9.1 & 13.7 & 0.9 & $<$ & 6.2 & $84 \pm 11$ & $98 \pm 2$ & $74 \pm 3$ & $5 \pm 2$ & $5 \pm 5$ & $12 \pm 4$ & 27 & 38 & 32 \\
\hline $\mathrm{H} 29$ & C. glabrata & 7.3 & 7.2 & 12.5 & 1.1 & $<$ & 7.2 & $84 \pm 9$ & $99 \pm 2$ & $75 \pm 1$ & $7 \pm 4$ & $3 \pm 2$ & $13 \pm 2$ & 27 & 36 & 31 \\
\hline $\mathrm{H} 4$ & C. parapsilosis & 7.2 & 16.9 & 17.6 & $<$ & $<$ & 0.4 & $4 \pm 4$ & $99 \pm 2$ & $2 \pm 1$ & $5 \pm 1$ & $0 \pm 0$ & $1 \pm 0$ & 11 & 7 & 9 \\
\hline H6 & D. rugosa & 7.2 & 17.5 & 17.9 & $<$ & $<$ & $<$ & $1 \pm 1$ & $66 \pm 5$ & $33 \pm 37$ & $16 \pm 6$ & $3 \pm 1$ & $6 \pm 1$ & 12 & 6 & 9 \\
\hline H8 & D. rugosa & 7.3 & 17.9 & 18.3 & $<$ & $<$ & $<$ & $3 \pm 2$ & $63 \pm 5$ & $31 \pm 33$ & $15 \pm 4$ & $5 \pm 5$ & $4 \pm 1$ & 12 & 4 & 8 \\
\hline H5 & D. rugosa & 7.3 & 17.8 & 18.1 & $<$ & $<$ & $<$ & $3 \pm 2$ & $67 \pm 8$ & $26 \pm 28$ & $10 \pm 2$ & $1 \pm 3$ & $3 \pm 1$ & 10 & 6 & 8 \\
\hline H9 & D. rugosa & 7.3 & 17.8 & 18.1 & $<$ & $<$ & $<$ & $2 \pm 1$ & $60 \pm 2$ & $29 \pm 29$ & $15 \pm 6$ & $5 \pm 4$ & $4 \pm 3$ & 11 & 4 & 8 \\
\hline $\mathrm{H} 7$ & D. rugosa & 7.4 & 18.0 & 18.4 & $<$ & $<$ & $<$ & $1 \pm 1$ & $60 \pm 5$ & $26 \pm 27$ & $10 \pm 2$ & $4 \pm 4$ & $4 \pm 3$ & 11 & 4 & 7 \\
\hline H323 & H. sp. & 10.2 & 16.7 & 22.8 & $<$ & $<$ & 5.5 & $10 \pm 12$ & $101 \pm 1$ & $16 \pm 22$ & $5 \pm 2$ & $34 \pm 23$ & $2 \pm 1$ & 25 & 26 & 25 \\
\hline H311 & $H$. sp. & 10.1 & 14.2 & 21.1 & $<$ & $<$ & 6.2 & $65 \pm 57$ & $98 \pm 2$ & $63 \pm 40$ & $7 \pm 5$ & $1 \pm 2$ & $1 \pm 0$ & 21 & 28 & 24 \\
\hline H369 & H. sp. & 7.6 & 4.5 & 7.2 & 2.1 & 0.2 & 9.5 & $85 \pm 28$ & $98 \pm 2$ & $58 \pm 46$ & $5 \pm 0$ & $2 \pm 2$ & $2 \pm 1$ & 23 & 25 & 24 \\
\hline H17 & H. opuntiae & 7.1 & 17.1 & 17.6 & $<$ & $<$ & $<$ & $32 \pm 55$ & $103 \pm 2$ & $16 \pm 22$ & $5 \pm 1$ & $1 \pm 1$ & $1 \pm 1$ & 15 & 31 & 23 \\
\hline H182 & H. opuntiae & 11.6 & 13.7 & 19.6 & $<$ & 0.2 & 10.8 & $8 \pm 11$ & $103 \pm 2$ & $39 \pm 10$ & $4 \pm 2$ & $3 \pm 2$ & $1 \pm 1$ & 15 & 26 & 21 \\
\hline H186 & H. guilliermondii & 11.2 & 5.5 & 11.4 & $<$ & 0.3 & 13.2 & $90 \pm 3$ & $103 \pm 1$ & $3 \pm 1$ & $5 \pm 2$ & $1 \pm 2$ & $2 \pm 1$ & 21 & 18 & 19 \\
\hline $\mathrm{H} 312$ & $H . \mathrm{sp}$. & 9.4 & 10.9 & 18.3 & $<$ & $<$ & 7.3 & $1 \pm 1$ & $101 \pm 3$ & $3 \pm 0$ & $4 \pm 1$ & $23 \pm 20$ & $2 \pm 0$ & 19 & 19 & 19 \\
\hline $\mathrm{H} 215$ & H. opuntiae & 9.6 & 18.6 & 20.1 & $<$ & $<$ & 3.8 & $31 \pm 54$ & $103 \pm 0$ & $3 \pm 1$ & $3 \pm 1$ & $1 \pm 2$ & $1 \pm 0$ & 15 & 19 & 17 \\
\hline $\mathrm{H} 201$ & H. opuntiae & 11.1 & 21.4 & 23.4 & $<$ & $<$ & 5.8 & $31 \pm 52$ & $101 \pm 0$ & $2 \pm 0$ & $3 \pm 1$ & $0 \pm 0$ & $1 \pm 1$ & 14 & 18 & 16 \\
\hline $\mathrm{H} 289$ & $M . \mathrm{sp}$. & 7.2 & 14.7 & 16.4 & 0.5 & $<$ & 2.0 & $14 \pm 13$ & $99 \pm 15$ & $1 \pm 0$ & $2 \pm 1$ & $0 \pm 0$ & $1 \pm 1$ & 12 & 11 & 11 \\
\hline
\end{tabular}

Growth \pm standard deviation (STD) was calculated from three biological replicate measurements of OD 600. The weighted average growth (Ø $\emptyset_{\text {Growth) }}$ was calculated using

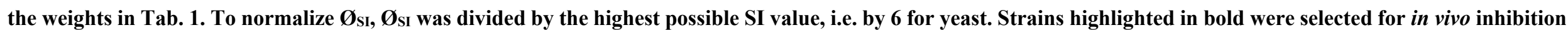

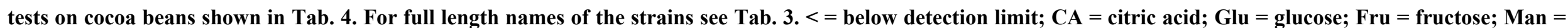

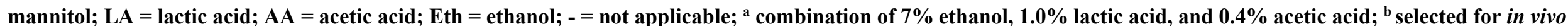
inhibition test as negative control strain 
Supplementary Table S4. Continued.

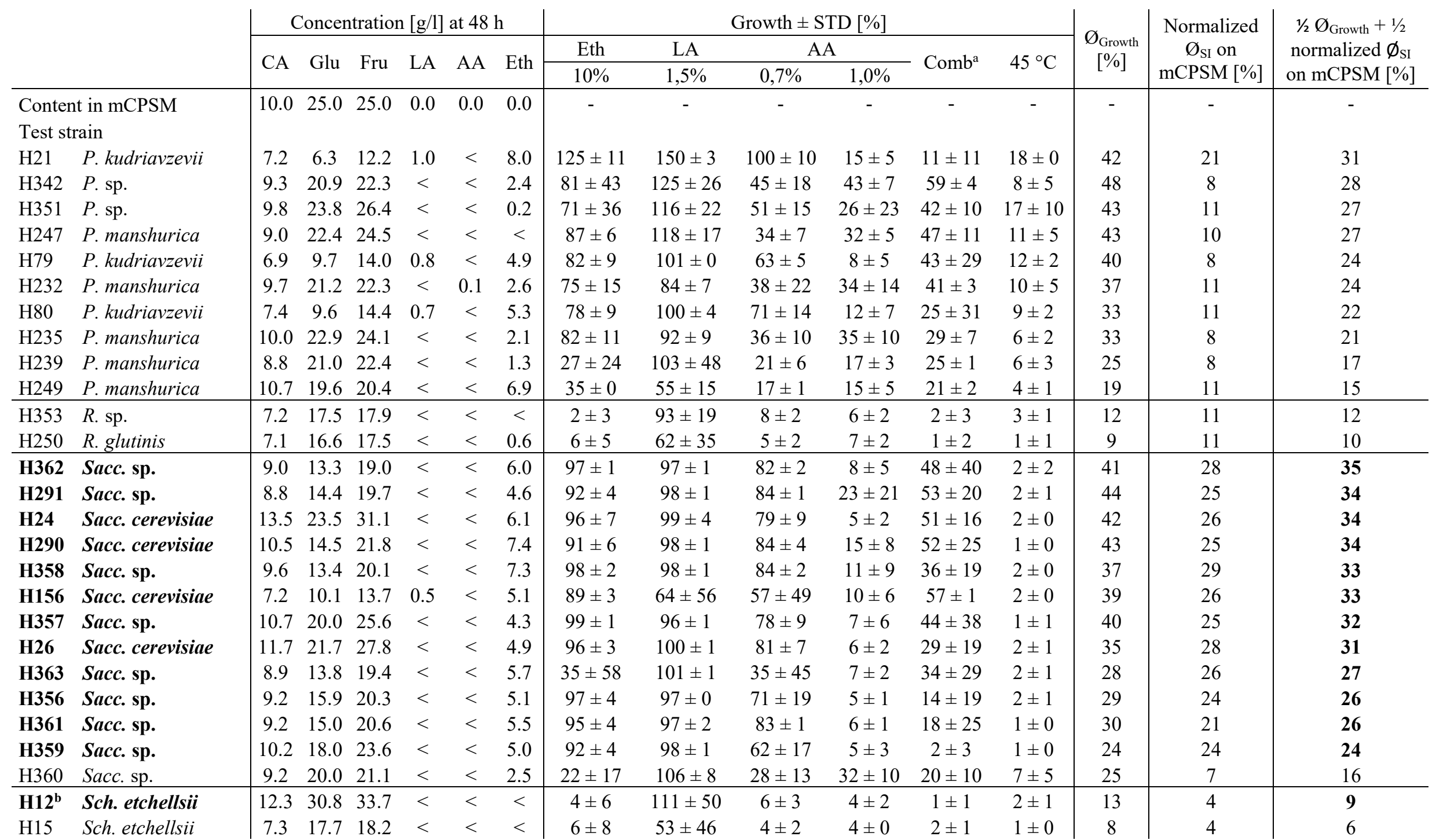

